\title{
Soils' seismic property research on the basis of investigation of their nonlinear properties
}

\section{B. Zaalishvili}

Geophysical Institute VSC RAS, Vladikavkaz, Russia

Received: 16 October 2014 - Accepted: 14 February 2015 - Published: 10 March 2015

Correspondence to: V. B. Zaalishvili (vzaal@mail.ru)

Published by Copernicus Publications on behalf of the European Geosciences Union \& the American Geophysical Union.

Solls' seismic property research on

the basis of investigation of their nonlinear properties

V. B. Zaalishvili

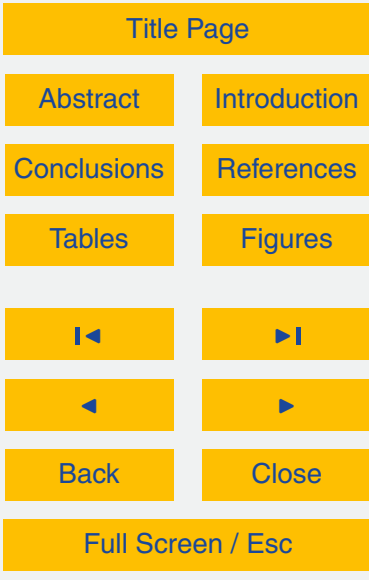

Printer-friendly Version

Interactive Discussion 


\section{Abstract}

The most important problem of seismic microzonation (SMZ) is to prove the assessment of soil conditions' influence on the response of buildings and constructions. On the modern stage, SMZ can not remain in "linear" positions, and empirical tools of

5 SMZ take into account nonlinear soil properties. A number of instrumental techniques for nonlinear phenomenon investigation in soils are given in the paper. New parameters describing nonlinearity are introduced and their practical usage efficiency is shown.

\section{Introduction}

Investigation of nonlinear phenomena in soils, which began in Russia nearly 50 years 10 ago, became the peculiar stimulus of modern investigations of the complex of geophysical indices, which are observed at strong and destructive earthquakes. These investigations have not only a scientific interest. It is economically and vitally important to predict soil and construction behavior (from the point of view of their adequacy for the expected seismic impact). The present investigation is the elaboration of the

\section{main scientific principles, which allow one to assess the nonlinearity of different soils} (on their lithologic compound and physical condition) for seismic microzonation (SMZ) purposes. Experimental and theoretical methods are used in the work to such an extent, which is necessary in order to develop soundly the physical foundations of the corresponding tools of the instrumental method of SMZ.

The most important problem of SMZ is to prove the assessment of this fact: how the soil conditions influence the reaction of buildings and constructions that underwent an intensive seismic impact. Engineering macroseismic observation of the territories, which are located in the epicenter zone of strong and destructive earthquakes, shows that real intensity effects often do not correspond to the expected results, which are obtained with the help of different SMZ methods. It is explained in many respects by a failure to take account of nonlinear phenomena, originating in soils at strong earth-

\section{NPGD}

2, 425-474, 2015

Soils' seismic property research on the basis of investigation of their nonlinear properties

V. B. Zaalishvili

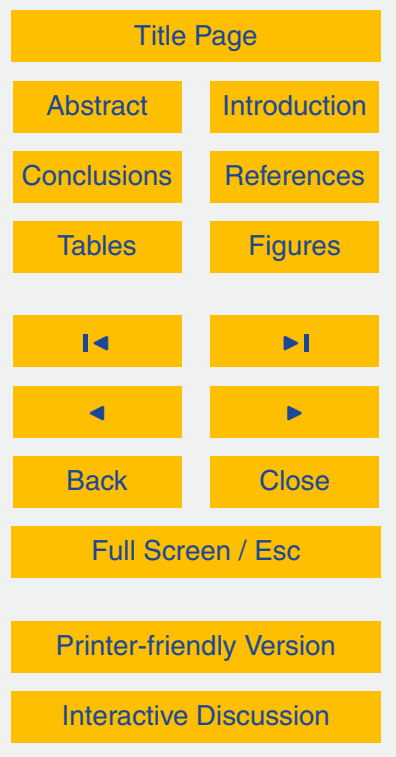


quakes. At the modern stage of the development, the SMZ can not remain in "linear" positions, although it must be noted that empirical tools of SMZ take into account (or must take into account) nonlinear soil properties. It lies in the essence of SMZ. Obviously, the question consists in the following: how exactly is the soil nonlinearity taken 5 into account and how impartial is this taking into account? The most adequate taking into account of nonlinearity (essential characteristics of natural phenomena) will allow one to approximate the corresponding anti-seismic measures to real features of seismic effects at strong earthquakes.

As a result of the fact that strong earthquakes are a rare phenomenon, the most 10 proven decision is the usage of the explosion tool from the SMZ instrumental tools. Moreover, even the presence of a strong earthquake unit record (which is of great value for SMZ) can not characterize the expected soil behavior, owing to the ambiguity, particularly, of the parameters of the expected impact. However, usage of the explosion tool is very limited: it is difficult for realization in urban territories.

15 The necessity of developing the SMZ tool (which allows one to assess soil influence on earthquake seismic effects), based on investigation of nonlinear soil properties with the help of modern non-explosive sources of high power, is obvious under such conditions.

The investigation of the SMZ method, which is based on the creation of intensive seismic vibrations using non-explosive impulse and vibration sources, allowed one to disclose simple and effective indices of soil nonlinearity, to develop the techniques of their assessment (on the basis of the existing records of strong and destructive earthquakes' study). The mechanisms of nonlinear phenomena effects, and their interconnection with the features of wave fields originating from different sources on the soil stratum surface, were investigated, and correlations between the intensity of the macroseismic effect and soil nonlinearity, which is observed at work with non-explosive sources, were determined for solving of the mentioned problems.

Thus, the methods, which allow one to assess the intensity increment of soils of typical areas in zoned territory, with taking into account of nonlinear soil properties, are

NPGD

2, 425-474, 2015

Soils' seismic

property research on

the basis of

investigation of their nonlinear properties

V. B. Zaalishvili

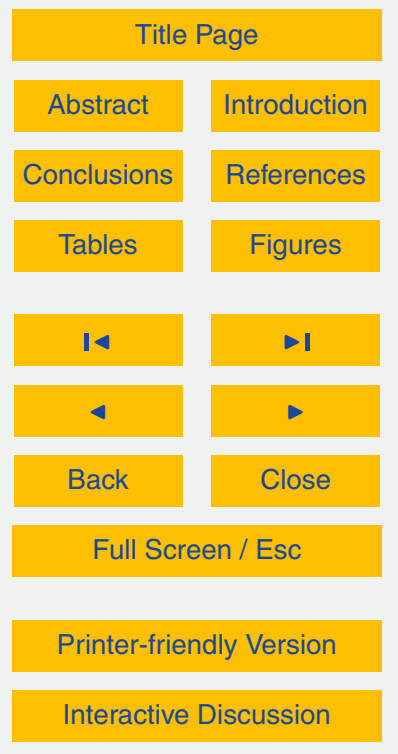


worked out and practically realized. For the first time on the basis of a nonlinear approach, the instrumental tools of SMZ, which allow one to assess and take into account (with intensity calculation) the degrees of nonlinearity and non-elasticity of soils, comprising the zoned territory, are obtained and approved; the indices of proper absorp5 tion and soil nonlinearity are introduced, based on the usage of connections between wave field spectral characteristics and the features of soil conditions; the universality of the introduced index characters is determined, which causes the opportunity of their usage in practical purposes; in order to calculate the intensity increment at strong earthquakes with taking into account of nonlinear transformations of wave fields close to a day surface, the following empirical formulas are introduced: (a) the formulas that use nonlinearity indices and elastic and non-elastic properties of the medium, and also (b) the formulas that connect the squares of normalized and real spectra with parameters of seismic impact (magnitude, acceleration, epicentral distance, duration and weight-average frequency of ground vibrations).

15 With the help of the developed tools, one can determine an intensity increment more exactly than with the help of the traditionally used tools, particularly, on dispersal (soft) soils, which are easily subjected to the external impact. The practice showed that the offered approach, which is, in essence, universal, can be used practically under any conditions (in cities nearby and in the territory of the responsible objects). And finally,

it is economically feasible to use the developed methods in order to assess the soil seismic properties (on the basis of the study of their nonlinear indices) with the help of modern powerful non-explosive sources.

Such features of the nonlinear approach show its prospects for SMZ. The main principles of the approach by usage of modern non-explosive sources with high power were the basis for the realization of SMZ works of a number of city territories of Georgia and Russia: Tbilisi, Rustavi, Gori, Tkibuli, Kutaisi, separate areas of the Bolshoi Sochi and, in addition, the territories of functioning and designed sites of the Novovoronezh atomic power plant (APP).

\section{NPGD}

2, 425-474, 2015

Soils' seismic

property research on

the basis of

investigation of their nonlinear properties

V. B. Zaalishvili

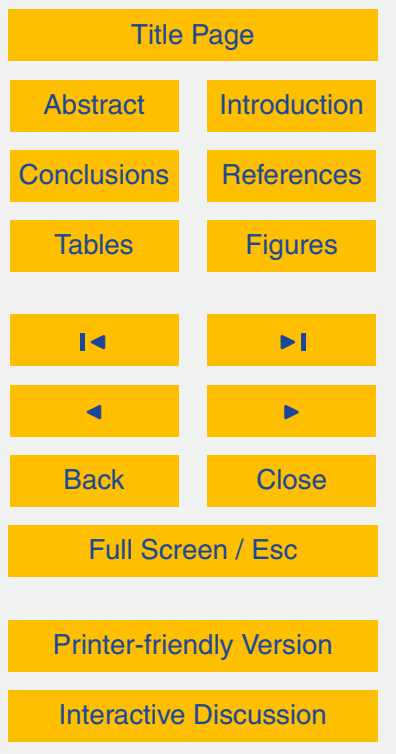


The results of the usage of the still improving approach show that it is highly efficient and practically feasible at SMZ to use modern non-explosive sources, which allows one to take into account nonlinear soil properties and considerably increase the efficiency of SMZ.

The used tools of the instrumental method of SMZ are based on the experience of the study of macroseismic effects at strong earthquakes and on the investigation of how seismic wave fields, which originated as a consequence of weak local and distant earthquakes, are distorted, depending on soil conditions, relief, and soil stratum structure.

10 Strong seismic motions, transformed from the original form under the influence of soil conditions, are studied on the basis of rough quantitative non-instrumental indices. The instrumental tools are used in order to assess features of weak movements of soil and to predict, with their help, the effect of strong earthquakes. A natural way of development of the instrumental method is by direct taking into account of nonlinear characteristics of soil immediately through the nonlinear distortions of a wave field, which is generated by the standard impulse or vibration sources. This caused the development of SMZ instrumental tools based on accounting of nonlinear soil properties using modern non-explosive sources. Modern mobile sources with high-impact stability allow one to obtain considerably more reliable results.

\section{Normalized and real spectra of vibrations}

The reasons for transformation of the shape and the spectrum of a seismic wave, connected with nonlinear soil conditions, can be represented in the following way.

The primary wave ( $P$ wave), incoming to the Earth's surface from the earthquake focus, undergoes a shape change in the top part of the cross section. These changes are connected with peculiarities of the structure and properties of the upper stratum of the sediments: the presence of a weathering zone, high absorption and nonlinear distortions.

\section{NPGD}

2, 425-474, 2015

Soils' seismic property research on the basis of investigation of their nonlinear properties

V. B. Zaalishvili

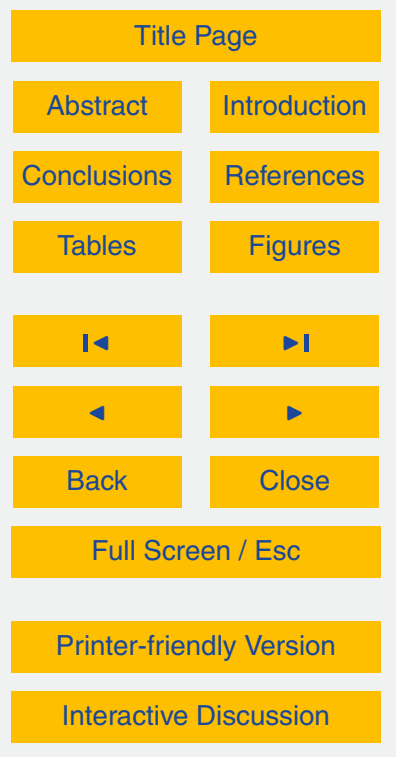


The weathering zone, which influences the seismic effect, has, as a rule, a different power (from several meters to 20-30 m), depending on geological conditions. The weathering zone influence on seismic wave amplitude can be calculated by means of geometric seismology laws taking into account the medium absorptive properties. This 5 influence is expressed by the incident wave amplitude and the spectrum change in consequence of resonance phenomena in the top stratum, which has maximal influence on the wave field forming at the Earth's surface, and also in subjacent strata, which have, as a rule, less influence on shapes of body waves.

Resonance phenomena are shown by the increase in seismic impulse duration and 10 the origin of maxima in their spectra at frequencies corresponding to constructive interference of the incident wave and the wave reflected from the day surface or from the bottom of the weathering zone. This condition is controlled by the ratio

$\lambda / 4=H$,

where $\lambda$ is the wavelength and $H$ is the thickness of the stratum.

15 Absorption in the soft top stratum of the soil is connected with the energy decrease in the high-frequency part of the spectrum. As a rule, this phenomenon is well taken into account by the exponential term

$\alpha=\exp (-\eta f H / V)$,

where $\eta$ is the decrement of the vibrations, $f$ is the frequency of vibrations, $H$ is the thickness of the strata, and $v$ is the propagation velocity of the wave.

In softer dispersed soils, the decrement of vibrations is $\eta=2-3$; therefore, significant changes in wave shape and its spectrum are observed in frequencies most of all:

$f>0.3-0.5 \mathrm{~V} / \mathrm{H}$.

For the boundary value of the longitudinal velocity $v=500 \mathrm{~m} \mathrm{~s}^{-1}$ for stratum $H=30 \mathrm{~m}$, the frequency of vibrations can be assessed:

$f \approx 0.3 \times 500 / 30=5 \mathrm{~Hz}$.
NPGD

2, 425-474, 2015

Soils' seismic

property research on

the basis of

investigation of their nonlinear properties

V. B. Zaalishvili

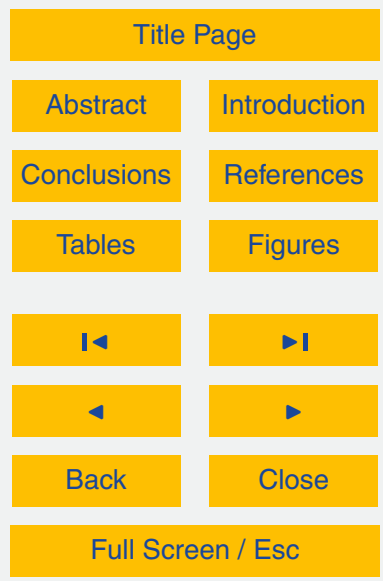

Printer-friendly Version

Interactive Discussion 
Above this frequency, the signal spectrum decreases quite extremely.

Both the resonance distortion of the wave and the absorption, considered here, are nonlinear processes, proceeding equally independently of the wave field intensity. Therefore, they can be assessed by means of seismic waves with low amplitude from 5 close and distant earthquakes and explosions. This property significantly distinguishes linear processes from nonlinear ones.

Nonlinear change in the waveform depends on its intensity; it intensifies at the increase in the seismic deformation level.

At the incidence of the intensive primary wave $P$ on the free surface, its detection 10 takes place: the phases of compression and dilatation are transformed in different ways by the soft medium, whereas elastic modules of compression and dilatation are different (it is easier to dilate the medium than to compact it). Such a medium can be described by a "stress-strain" diagram, which has a breakpoint in zero and consists of two lines. This medium is referred to as "bi-modular". This model of soft rock was offered 15 by Nikolaev (1967). It was experimentally confirmed by A. Gvozdev and V. Kuznetsov in 1977. A considerable distortion of the seismic signal during its propagation through the bi-modular medium can be clearly observed in the thin surface soil stratum.

Let us imagine an intensive seismic primary wave falling on the surface. In the depth $(H)$ of several and more tens of meters the component of dilatation, transferred by the wave, does not exceed lithostatic stress $\rho \mathrm{gH}$ and only realizes partial unloading. At that the "two-modularity" of the medium is not observed, as the elastic modules, corresponding to compression, are predominant. At less depth, when dilatation exceeds $\rho \mathrm{gH}$ in its value, the realization of the elastic deformations takes place at the elastic modules of dilatation, which are smaller than the modules of compression.

Nonlinear distortions of the wave shape, which take place in the bi-modular medium, occur in the spectrum "spreading" to the areas of low and high frequencies (Nikolaev, 1967; Vasilyev et al., 1969; Trifunac, 1994). The energy transition to the low-frequency area is connected with modulation, difference in character of soil movement up and down as a result of different modularity. Constant deformation of medium volume di-

\section{NPGD}

2, 425-474, 2015

Soils' seismic

property research on

the basis of

investigation of their nonlinear properties

V. B. Zaalishvili

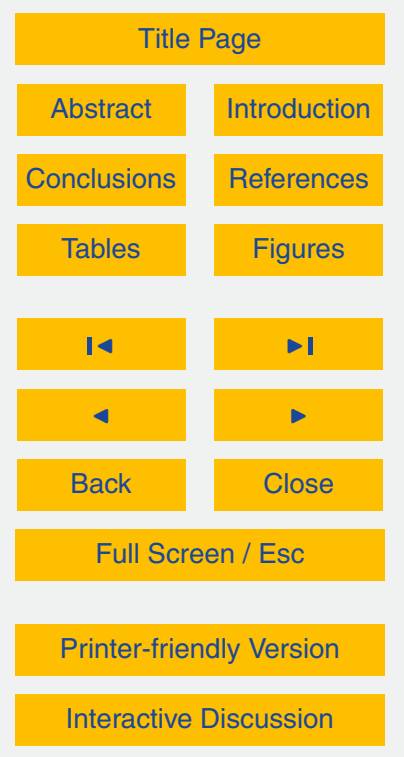


latation of intensive vibrations takes place. It is called a "seismic-radiative" deformation (Vasilyev et al., 1987). At registration by a seismograph, which is only sensitive to the variable field, constant deformation is not registered. At the same time the effect of the spectrum saturation by low frequencies is obvious. The spectrum saturation by high 5 frequencies is connected with both the effect of detecting and the nonlinear interaction and self-interaction of seismic waves (Nikolaev, 1987; Engelbrecht and Feldman, 1987; Beresnev et al., 1987). Earlier attention has also been paid to the opportunity of spectrum saturation by high-frequency components (Mandelshtam, 1950).

Thus, the spectrum spreading of the seismic impulse of the longitudinal wave, which 10 takes place at strong seismic deformations, is an indicator of the nonlinear wave shape distortion. This phenomenon accompanies the change in the primary seismic wave shape and chaotic interference vibrations. The spectrum width is a simple quantitative measure of this process and of nonlinear soil properties, accordingly.

Actually, let us imagine that the initial spectrum (i.e., the spectrum undistorted by 15 nonlinear processes) has a pronounced resonance shape (Fig. 1a). The signal distorted by nonlinear phenomena is characterized by the increment of the spectrum square (Fig. 2b) or expansion.

It is clear that the increase in vibration frequencies $\Delta f$ depends on seismic wave intensity. In order to bring all the measures to equal intensity, the way of standard seismic source usage (vibration or impulse non-explosive sources) and the way of standard conditions of measures have been chosen. The modern Russian vibrator equipment SV-10/100 initiates seismic vibrations with an intensity of approximately 7 points in the epicentral zone. At such an intensity, the above-mentioned nonlinear processes (whose quantitative measure can be the width of the normalized spectrum wave range) occur in full measure.

In spite of the fact that this characteristic is a sufficiently rough representation of the measure of nonlinear seismic properties and nonlinear transformation of seismic signals, nevertheless, it is quite acceptable at the first stage of investigations, when the main quantitative ratios are determined. The efficiency of this quantitative measure

\section{NPGD}

2, 425-474, 2015

Soils' seismic

property research on

the basis of

investigation of their nonlinear properties

V. B. Zaalishvili

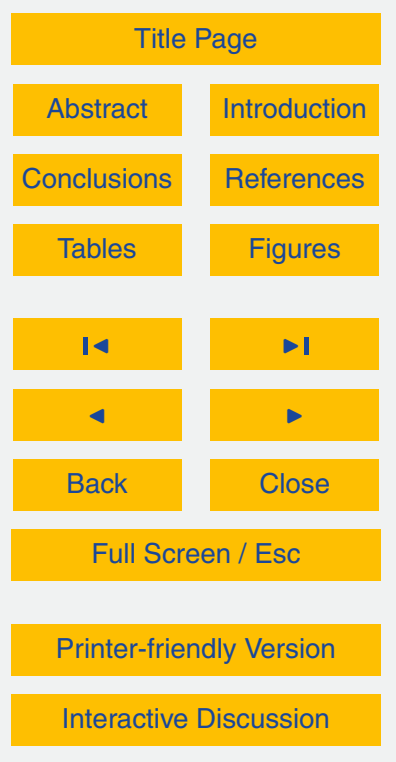


usage demands experimental checkout of the empirical formulas, made on the basis of normalized spectrum width at those sites, where the real seismic effect of strong earthquakes is known.

On the other hand, the analysis of the accelerogram of the Racha earthquake and 5 the corresponding vibration spectra (amplitude Fourier spectra) has shown that a very interesting parameter with its correlation properties is singled out: a normalized spectrum of ground vibrations and, to be more precise, the square of the area under the spectral distribution curve of the spectrum, scaled on a maximal amplitude or peak (Fig. 2).

10 Thus, the square of the normalized spectrum is

$S_{\mathrm{N}}=\frac{1}{A_{0}} \sum\left(A_{i} f_{i}\right)$

where $A_{0}$ is the maximal amplitude of the spectral distribution curve, and $A_{i}$ and $f_{i}$ are the amplitude and the frequency of the $i$ component of the spectrum.

Because of the fact that the value of the ordinate remains constant for all the consid15 ered cases, the square of the normalized spectrum will be proportional to the number of separate components; i.e., it characterizes the "extension" of the spectrum, its width.

In the result of processing of the data, registered in areas of different lithologic structures of soils, the dependence of the normalized spectrum squares $S_{N}$ on acceleration has been received. The data were approximated by a broken line and also in the form of a polynomial of $n$ order on the basis of mean-square deviation and other criteria (Dzhindzholava, 1986; Leman, 1964). $S_{N}$ parameter changes most monotonously in the data records of station Ambrolauri. The character of changing for the given dependence for records of station Oni differs extremely (for all three components) from similar dependences for the other areas (Fig. 2). It must be noted that station Oni was located 25 on rocky soil.

As has already been noticed, the acceleration value has a wide application in various types of engineering seismology analyses and the seismic stability theory. At the same

\section{NPGD}

2, 425-474, 2015

Soils' seismic property research on the basis of investigation of their nonlinear properties

V. B. Zaalishvili

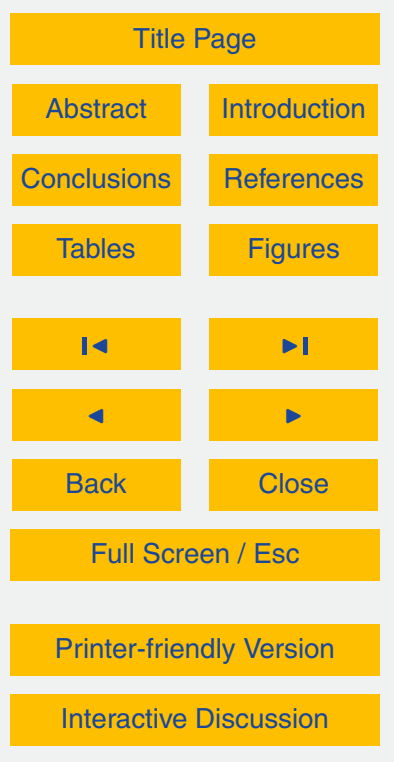


time, the bad correlation of acceleration with emitted seismic energy inversely related to the frequency of vibrations (easily determined in the record) is often quite known. It sets conditions for the investigation of the dependence of vibration frequency on acceleration. A very complex character of the given dependence for separate areas 5 must be noted (stations Zemobari and Iri).

Thus, the conclusion about the possibility of high frequencies of vibrations for both small and large acceleration values is representative (Fig. 2). Taking into account that, as a rule, the frequencies themselves are very closely connected with magnitudes, the ambiguity of correlation between magnitude and acceleration is explainable. At high 10 acceleration indices, vibration frequency dependences are similar for rocky and soft soil.

Thus, at small initial values of accelerations, the maximal vibration frequency sharply decreases with acceleration increase. At that, low-frequency content is usual for vibrations (Fig. 2). Thereafter (with acceleration increase), the growth in the corresponding 15 vibration frequency is observed. This fact explicates the information known in the literature - that at small frequencies, the acceleration has a larger impact on a construction (i.e., the level of the acceleration amplitude is high enough here) and the absorbing of energy is lowest, as the vibration frequency is small. "Average amplitude levels" of accelerations may cause large damage of relatively flexible buildings, whereas highfrequency accelerations, which exceed some "critical area" of their values, rapidly diminish due to high absorption. Obviously, observed small damages at high-frequency vibrations are sometimes explained by this fact.

Actually, due to the highest absorption in surrounding soft soils, a hazardous amplitude level for a construction cannot simply be reached. This explains the fact that building damage is minimal on the soils with high acceleration values. At Niigata, earthquake accelerations were small (soft sandy soils, which are characterized by very high absorption for high frequencies, are present).

Thus, the greatest impact on soil is realized at low frequencies corresponding to high magnitudes or energies.

\section{NPGD}

2, 425-474, 2015

Soils' seismic

property research on

the basis of

investigation of their nonlinear properties

V. B. Zaalishvili

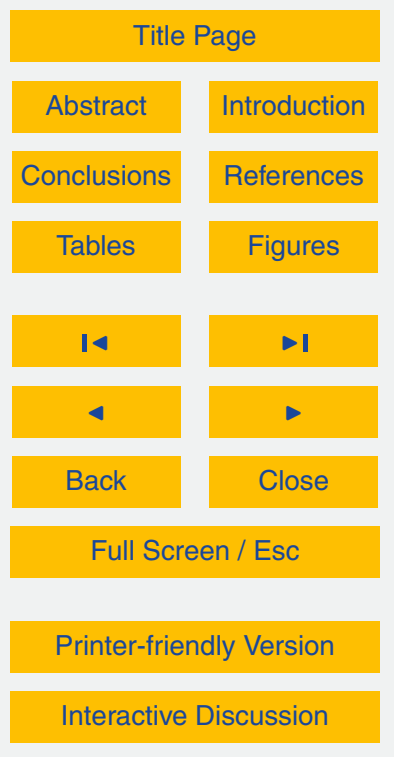


Dependence is correlated better, if, at the analysis, the average weighted value of maximal frequencies is used instead of maximal frequencies. It is calculated using the formula (Zaalishvili, 1987)

$f_{\mathrm{aw}}=\sum A_{i} f_{i} / \sum A_{i}$

5 where $A_{i}$ is an amplitude and $f_{i}$ is the corresponding frequency of the vibration spectra.

The weighted average values of the periods of the spectra correctly (from the physical point of view) reflect the stress-strain state of the vibrating soil stratum. Dispersion of values for soft soils becomes far less. The considered dependence of vibration frequency, as a normalized spectrum square for loose soils, decreases practically linearly with acceleration (at their small values), and at defined values (determined for each soil type and its condition), for example, $0.08-0.12 \mathrm{~g}$, a nonlinear change in dependence takes place.

The analysis of the given dependences shows that squares of the normalized spectrum of ground vibrations are proportional to the average weighted values of the corresponding frequencies.

So, the higher the weight-average frequency is, the more normalized the spectrum square is.

In other words, the normalized spectrum square $S_{N}$ of vibrations, characterizing the distribution of the given energy of the earthquake according to the spectrum components, is nothing more than an indicator of the absorption value of this energy. At small values of accelerations, $S_{N}$ has a sufficiently sizable value for soft soils (Fig. 2).

With further growth of acceleration, the $S_{N}$ value decreases and reaches some limit value, caused by the capability of the system to absorb energy. After reaching the limit value, the absorption grows again with acceleration increase. The complex character of the dependence is, obviously, explained by the absorption ambiguity on strong and weak dynamic impacts. Different accelerations can correspond to one frequency value. It must be also noted that, as each point on given dependences is the "result" of an individual earthquake impulse with its own value, epicentral distance, depth of focus,
NPGD

2, 425-474, 2015

Soils' seismic property research on the basis of investigation of their nonlinear properties

V. B. Zaalishvili

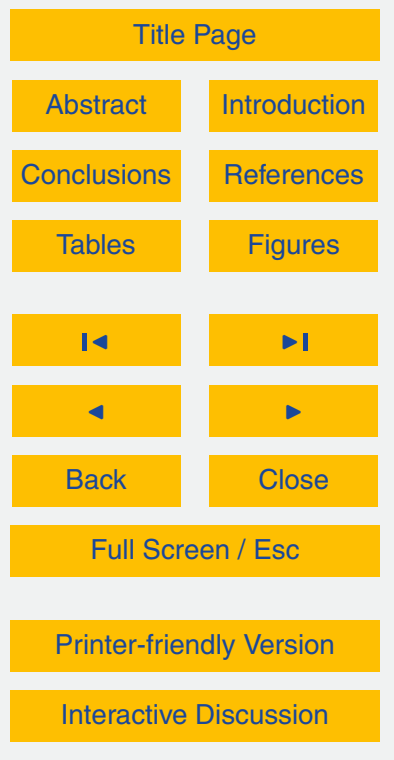


etc., we must speak not about bad correlation, but about very unexpected small data scattering. Therefore, we can come to a conclusion about the high content richness of the considered parameter.

The $S_{N}$ value has a frequency dimension and represents the width of the wave range 5 of seismic vibrations. Due to the frequency-selective character of absorption and the complex frequency-dependent character of nonlinear distortions of seismic vibrations, the value of the normalized square of vibrations $S_{N}$ characterizes the development of both processes.

At the same time, the width of the wave range gives an insufficient conception of the predominant frequencies of the record and their dynamics connected with the evolution of the wave shape at its propagation in a real medium. As the result of this, one more parameter - the "weight-average frequency" of vibrations - is used.

Substantially, three parameters of the spectrum, $A_{0}, S_{\mathrm{N}}$ and $f_{\text {aw }}$, are its major characteristics. The evolution of even these greatly averaged parameters in the top part of the 15 sedimentary stratum occurs in a complicated manner, and it is affected by absorption heterogeneity and medium nonlinearity.

In SMZ problems, changes in seismic wave fields at relatively small distances on the order of fractions of the wavelength are considered. Therefore, we may neglect the influence of intensity change in consequence of geometric divergence.

The influence of large and average heterogeneities, which have a size on the order of the wavelength and more, is taken into account by differential assessments of vibration intensity on different types of soils. The influence of small heterogeneities, which have a size on the order of wavelength fractions, is not usually taken into consideration.

The features of changes in the seismic wave spectra, connected with their absorption

and nonlinear distortion, are generally formed in the top thin soil layer, varying from several to a dozen meters. Even at high linear absorption at such distances, the energy loss on absorption is relatively moderate. High frequencies have a significantly higher absorption than low frequencies; the spectrum decrease by high frequencies occurs
NPGD

2, 425-474, 2015

Soils' seismic

property research on

the basis of

investigation of their nonlinear properties

V. B. Zaalishvili

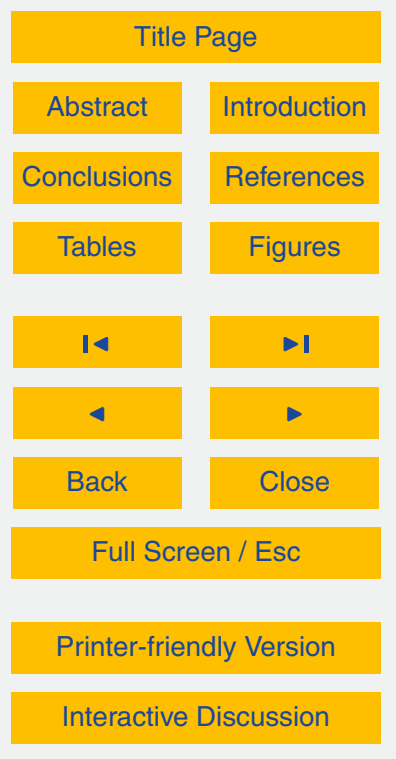


and, as a result of that, the width of the $S_{\mathrm{N}}$ band, the weight-average frequency $f_{\text {aw }}$ and the vibration amplitude $A_{0}$ increase.

The nonlinearity influence on the change in the spectrum characteristics of seismic vibrations is considerably different.

$5 \quad$ Ideally, at weak soil nonlinearity in propagating harmonic waves, the form distortion appears, which can be characterized by the growth in the amplitude of the second harmonics $A_{2}$ relative to the amplitude of the major tone $A_{1}$ :

$A_{2} / A_{1}=M^{\prime} K r$,

where $M^{\prime}=2 \pi f_{\mathrm{aw}} A_{1} / V_{\mathrm{s}}$ is the Mach number, $r$ is the distance, and $K$ is the nonlinearity 10 factor.

Thus, the nonlinear transformation of the wave shape happens sufficiently smoothly with a distance, and even at large values of $K$ and $M^{\prime}$, these changes will be relatively small. The spectrum spreading to the high-frequency region takes place due to the aliquot harmonics origin. In many cases, nonlinear distortions also appear in the form of the sub-harmonic of low-frequency harmonic vibrations; the given process is accompanied by the spectrum spreading to the low-frequency region.

So, at propagation through the elastic-nonlinear medium, the spectrum spreading of the seismic wave, the increase in $S_{\mathrm{N}}$ and $f_{\text {aw }}$ values, and the decrease in $A_{0}$ take place. In order to take into account the seismic energy distortion in the top part of the soil stratum, the theory of distorted damping is used. According to the given theory, the loss coefficient $\eta$ (the value that is reverse to the $Q$-factor) is equal to the ratio of the spectrum width and the resonance frequency (in many practical cases, the resonance frequency is close to the weight-average frequency; the spectrum width is a normalized spectrum square):

${ }_{25} \eta \approx S_{\mathrm{N}} / f_{\text {aw }}$.

The loss coefficient increases with the deformation growth; therefore, the seismic energy absorption is also a nonlinear process. At short distances of intensive seismic 437
NPGD

2, 425-474, 2015

Soils' seismic property research on the basis of investigation of their nonlinear properties

V. B. Zaalishvili

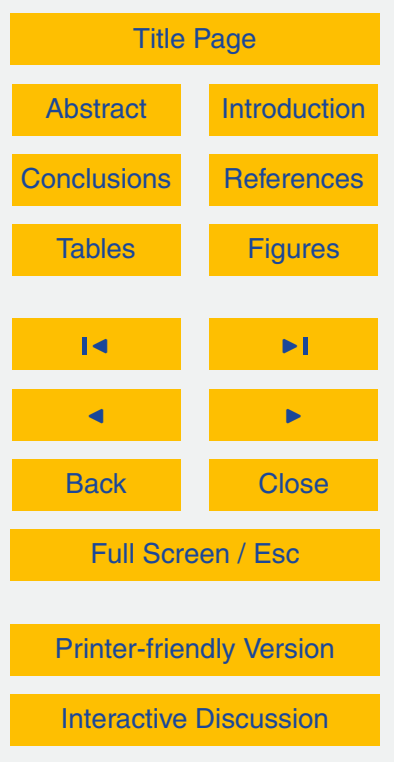


wave propagations, the role of absorption in the wave field change is less than the nonlinearity role, which is sharply displayed at the surface of a loose medium.

A change in the deformation sign at wave propagation through a bi-modular medium must be accompanied by specific effects: at that, both primary and shear waves un5 dergo (experience) a sharp change in drift velocity and acceleration velocity, as elastic deformation energy must be adapted to the new modules during short periods in comparison with a typical vibration period. In vibration processes, this will be expressed in the form of rapid spreading of the spectrum width to both high-frequency (HF) and low-frequency (LF) directions and also in the appearance of the constant component 10 (constituent). Intensive HF impulses will originate in longitudinal and transverse vibrations of soils; parameters $A_{0}$ and $f_{\text {aw }}$ sharply increase.

On solving the $S M Z$ problem, the comparative intensity change at one site relative to another reference site is assessed. Each individual assessment of intensity depends on the epicentral distance, Earth's crust structure on the route of the focus-observation 15 point of seismic wave features, emitted by the focus. The most consistent relative assessments of intensity differences conform to the cases when the compared observation points are at relatively short distances from one another, so the factor of geometric deviation and wave field distortion by large medium heterogeneities, located on the path from the source to the surface, makes an equal contribution to the seismic effect 20 formation.

The width of the spectral band is connected by simple ratios with the seismic parameters of soil, such as the transverse wave velocity $\left(v_{\mathrm{S}}\right)$, the coefficient of absorption $(\alpha)$ and the vibration decreement $(\eta)$. At the propagation distance $r$, the energy increase is assessed by the exponent $\exp \left(-\eta f_{\mathrm{aw}} r / v_{\mathrm{S}}\right)$, and then

${ }_{25} \eta f_{\mathrm{aw}} r / V_{\mathrm{s}}=\eta r / \lambda=\alpha r$.

In the frequency range $0.5-30 \mathrm{~Hz}$ for sedimentary soils, it can be supposed that $\alpha$ is proportional to $f_{\mathrm{aw}}$. At small exponent indicators, the absorbed energy is proportional to $\alpha r$. Taking into account the hysteresis character of attenuation, we get the ratios
NPGD

2, 425-474, 2015

Soils' seismic

property research on

the basis of

investigation of their nonlinear properties

V. B. Zaalishvili

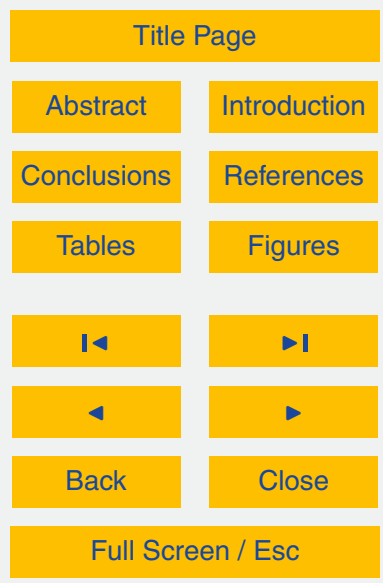

Printer-friendly Version

Interactive Discussion 
$S_{\mathrm{N}}=\eta f_{\mathrm{aw}}^{2} r / v_{\mathrm{S}}$,

$S_{\mathrm{N}} / f_{\mathrm{aw}}=\eta f_{\mathrm{aw}} r / v_{\mathrm{S}}=\alpha r$.

For some chosen distance ( $r=$ const), it can be recorded as

$S_{\mathrm{N}} / f_{\text {aw }} \sim \alpha$.

5 Thus, the quantity of the ratio between the normalized spectrum and the frequency is directly proportional to the energy absorption by the given stratum (Zaalishvili, 1996).

It is interesting to consider the dependence of a "pure" absorption value on acceleration (Fig. 3). It is obvious that at the initial stage, i.e., at low influence, the absorption increases with influence growth, but after acceleration $a=0.08 \mathrm{~g}$, absorption begins to

10 decrease, and reaches a minimum at acceleration $a \approx 0.2 \mathrm{~g}$. On further acceleration, the growth absorption index increases again, but with less velocity. Similar curves are obtained for other areas formed by soft soils (stations Zemobari, Iri, etc.).

On the other hand, at nonlinear soil response the spectrum spreading to the HF and partly to the LF spectrum regions is typical. The spectrum spreading to the LF regions, caused by the absorption phenomena, in soft soils, exceeds the spreading connected with soil nonlinearity, and so the absorption "masks" nonlinearity. In rocks where the absorption value is much lower, the medium nonlinearity appears more distinctly. At the same time, the assessment of nonlinearity from the point of SMZ view is interesting exactly for soft soils. Moreover, at usage of the normalized spectrum square, the opportunity to study the spectrum shape is absent. In connection with the information mentioned above, it is necessary to introduce another index in order to assess the nonlinearity degree of soils.

In this connection, the concept of a "real" vibration spectrum square has been introduced. It is a "vibro-spectrum" or an ordinary amplitude spectrum of Fourier, calculated on the seismogram. The real spectrum square $S_{r}$, according to the definition, is equal to

$S_{\mathrm{r}}=S_{\mathrm{N}} A_{0}$
Soils' seismic property research on the basis of investigation of their nonlinear properties

V. B. Zaalishvili

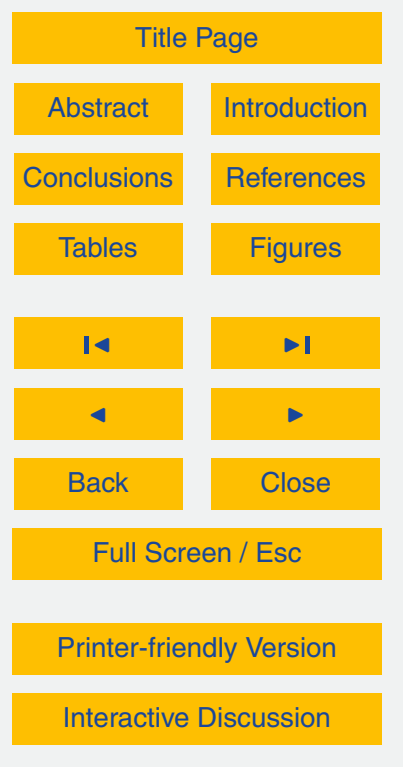


The analysis of earthquake instrumental records shows that the value $S_{\mathrm{p}}$ is in close connection with soil nonlinearity. At reaching accelerations $a \approx 0.1 \mathrm{~g}$ (Fig. 4), the dependence of $S_{\mathrm{p}}$ on acceleration changes sharply assumes a nonlinear character ("soft nonlinearity"). For rigid soils, "rigid" nonlinear dependence is typical.

$5 \quad$ Taking into account Eq. (11), Eq. (10) will take on the following form:

$S_{\mathrm{r}} \sim \alpha A_{0} f_{\mathrm{aw}}$.

Thus, the real spectrum square $S_{p}$ is an integral characteristic of absorption and nonlinearity phenomena, which appear in soil strata. It confirms the similarity of absorption and nonlinearity phenomenon influence on the spectrum shape observed at experi10 ments.

The ratio of the real spectrum square and the absorption will obviously give the socalled "pure" nonlinearity:

$S_{\mathrm{r}} / \alpha \sim A_{0} f_{\mathrm{aw}}$.

As has already been noted, the absorption in soft soils is directly proportional to the 15

$S_{\mathrm{r}} / f_{\mathrm{aw}} \sim A_{0} f_{\mathrm{aw}}$.

At that, the characteristics of soil nonlinearity can be directly assessed using Eqs. (13) or (14). For practical purposes, the usage of the latter relation is evidently more preferable.

20 So, the spectrum amplitude and the weighted average frequency product, being the simplest and most easily measured value, characterizes nonlinearity - a new quality of soil. In other words, new indices, which are in close connection with direct indices of absorption and soil nonlinearity, have been introduced. In contrast to the traditional indices, the new ones are directly measured on the spectra of ground vibrations.

\section{NPGD}

2, 425-474, 2015

Soils' seismic property research on the basis of investigation of their nonlinear properties

V. B. Zaalishvili

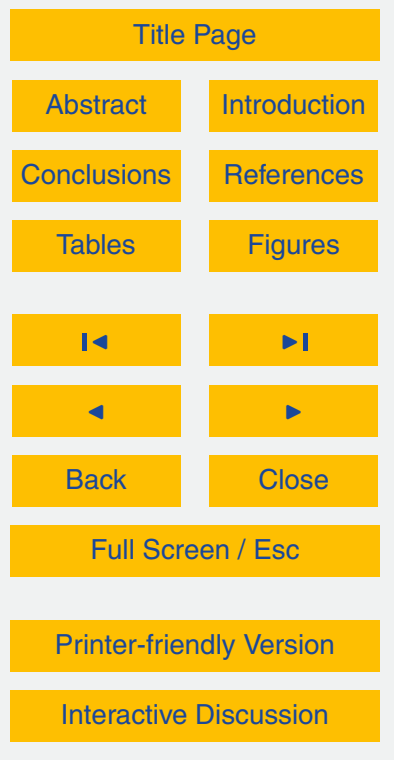




\section{Seismic microzonation based on accounting of nonlinear soil properties}

It is shown in the chapter that the square of the vibration spectrum is quite an important index of soil properties and any physical system.

On the consideration of Eq. (12), one can conclude that at a constant value of non5 linearity of the given soil (i.e., at $A_{0} f_{\text {wa }}=$ const), absorption increase will lead to the proportional increase in the real spectrum square. Indeed, the softer the soil is, the more it absorbs the introduced energy, and the larger the square of the real spectrum of its vibrations is. In rocks where the absorption is low, the square of the real spectrum is small.

10 For a more accurate definition of seismic properties of soils, the concept of an "area of under-spectral region of the vibration spectrum" of soils was introduced. To calculate the intensity increments, a method of vibration spectrum was developed, and the following formula was proposed (Zaalishvili, 1986):

$$
\Delta l=2 \lg \frac{S_{\text {ri }}}{S_{\text {ro }}}
$$

15 where $S_{\mathrm{ri}}$ is the area of a real vibration spectrum of the investigated soil, and $S_{\mathrm{ro}}$ is the area of a real vibration spectrum of the reference soil.

When comparing the absorption and nonlinearity indices with the conformable spectra of ground vibrations, it was found that, at high absorption indices, the spectrum square predominates in the LF field and, at high nonlinearity, it predominates in the 20 HF field of the spectrum. Otherwise stated, the existence of absorption is reflected in additional spreading of the LF region of the spectrum, and the existence of nonlinearity in the spreading of the HF range.

Aforesaid allowed one to obtain the formula for calculation of the intensity increment on the basis of taking into account nonlinear elastic soil behavior or elastic nonlinearity 25 (on usage of a vibration source) (Zaalishvili, 1996):

$\Delta /=3 \lg A_{i} f_{\text {awi }} / A_{0} f_{\text {awo }}$,

\section{NPGD}

2, 425-474, 2015

Soils' seismic property research on the basis of investigation of their nonlinear properties

V. B. Zaalishvili

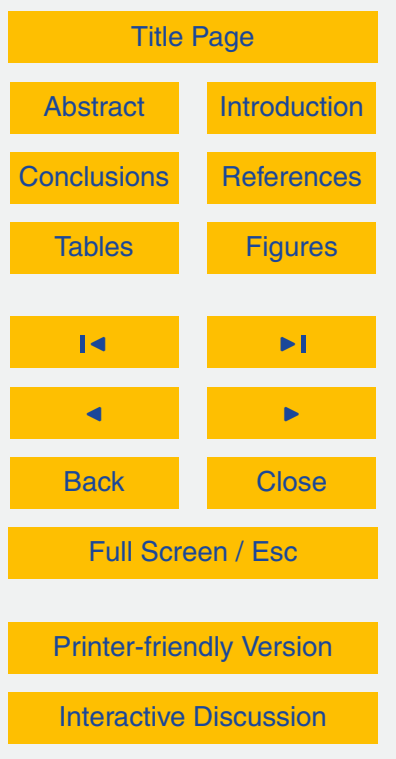


where $A_{i} f_{i}$ and $A_{0} f_{0}$ are the products of the spectrum amplitude at the weighted average vibration frequency of investigated and reference soils, respectively.

Eq. (14) describes soil nonlinear-elastic behavior at the absorption absence. In the case of the impulsive source usage at SMZ, the formula will be as follows (Zaalishvili 5 and Timoshuk, 1996):

$\Delta /=2 \lg A_{i} f_{\text {awi }} / A_{0} f_{\text {awo }}$.

Equations (15) and (16) were applied at SMZ of the Kutaisi city territory and also at SMZ of the territory of Novovoronezh APP-1 and the site for designed APP-2.

It must be noted that, in comparison with Eqs. (15) and (16), Eq. (14) describes the soil nonlinear-elastic behavior at absorption presence (Zaalishvili, 2012).

In addition, we carried out special investigations in order to determine directly the soil nonlinearity index in the form of their strain sensitivity (Fig. 5).

The investigations were held in the region of Gori in the territories of the villages of Barbalaant Kari and Metekhi in direct nearness from two churches, which survived 15 the destructive Kartli earthquake (20 February 1920). The churches of St. George are located on equal distance from the earthquake epicenter on the top of hills with equal elevation. The constructions themselves, which are represented by a one-nave basilica with the walls, made of ashlars on whitewash, are single type in the constructive respect. The first church, situated on the loam stratum (of semisolid consistency) with 20 a depth of more than $15 \mathrm{~m}$, was destroyed as a result of the mentioned earthquake. The second church (Metekhi), which was situated on pebble stratum (conglomerate) with a depth of more than $15 \mathrm{~m}$, practically was not damaged. In order to assess the soils' nonlinearity indices, their strain sensitivity, which characterizes the change in soil stratum properties at the change in pressure on it, was investigated. $P$ wave propagation velocity in soil was considered as varying parameter. Experimental investigations consisted in the following. On the both sides of the plate of seismic vibration source SV-10/100 the sensors of ultrasonic flaw detector UK-14P and $P$ wave propagation velocity $V_{\mathrm{P}}$ was measured. The coefficients of soil strain sensitivity that characterize

\section{NPGD}

2, 425-474, 2015

Soils' seismic

property research on

the basis of

investigation of their nonlinear properties

V. B. Zaalishvili

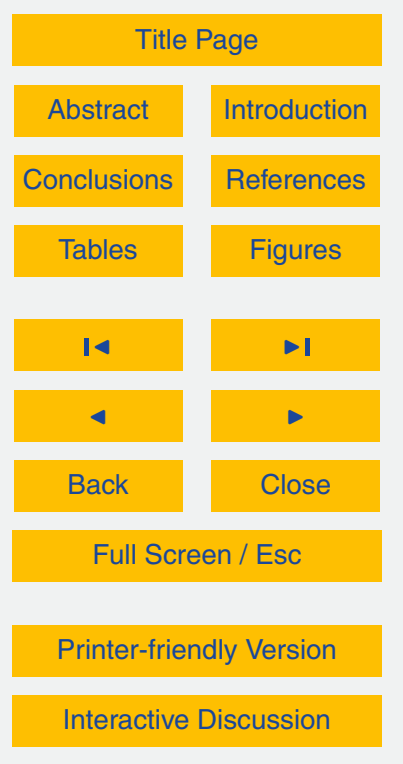


the physical nonlinearity in the form of $N(K \approx 0.56 N)$ were calculated by the special formula (Nikolaev, 1987)

$$
K=\rho V(\Delta V / \Delta P)
$$

where $K$ is the coefficient of soil strain sensitivity, $\Delta V$ is the change in $P$ wave velocity, 5 and $\Delta P$ is the pressure change.

For strain sensitivity calculations, the pressure change was taken as $\Delta P=0.08 \mathrm{MPa}$. Weighted average values $\Delta V_{1}$ and $\Delta V_{2}$ of changes for clay (soil density $\rho_{1}=1.8 \times$ $\left.10 \mathrm{~kg} \mathrm{~m}^{-3}\right)$ and macro-fragmental soils $\left(\rho_{2}=2.1 \times 10^{3} \mathrm{kgm}^{-3}\right)$ were 50 and $5 \mathrm{~m} \mathrm{~s}^{-1}$, respectively.

10 A notable difference in the intensity effect $(\Delta /=1)$ made it possible to obtain an expression for the calculation of the increment of seismic intensity:

$\Delta /=3 \lg \frac{K_{1}}{K_{2}}$,

where $K_{1}$ and $K_{2}$ are strain sensitivity coefficients.

By substituting values of the coefficients into Eq. (19), we obtain (Zaalishvili, 1996)

$\Delta /=3 \lg \frac{\rho_{1} V_{1} \Delta V_{1}}{\rho_{2} V_{2} \Delta V_{2}}$

where $\rho_{1} V_{1}$ and $\rho_{2} V_{2}$ are the seismic rigidities of the compared soils, and $\Delta V_{1}$ and $\Delta V_{2}$ are the velocity changes in compared soils stipulated by their strain sensitivity.

It should be noted that the resulting ratio, despite the form similarity to well-known traditional SMZ methods of seismic rigidities, according to internal content, differs significantly. So, here explicitly in the formula of the intensity increment, "nonlinear" member $\Delta V$ is included. The introduction of this parameter allows us to differentiate the seismic properties of the column in its degree of deviation from the linear behavior.
NPGD

2, 425-474, 2015

Soils' seismic property research on the basis of investigation of their nonlinear properties

V. B. Zaalishvili

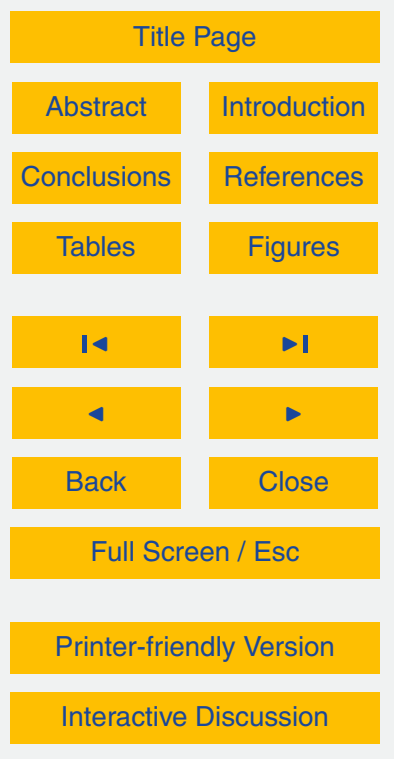


In conclusion, let us return to the evaluation of nonlinearity and compare it with an estimate of the nonlinearity in Eq. (20), the resulting assessment of the phenomenon of strain sensitivity.

Let us take our mind off the intensity increments or intensity, assessing the "under5 logarithmical" value in Eq. (20):

$\frac{\rho_{1} V_{1} \Delta V_{1}}{\rho_{2} V_{2} \Delta V_{2}}=\frac{1.8 \times 10^{3} \times 200 \times 50}{2 \times 1 \times 10^{3} \times 800 \times 5}=2.12$

On the other hand, accounting that the experimental studies of strain sensitivity were performed at the same sites where the features of the spectra were studied using the appropriate vibro-spectra parameters, finally, one can obtain

10 $\frac{A_{1} f_{1}}{A_{2} f_{2}}=\frac{0.8 \times 30}{0.2 \times 55}=2.18$.

Thus, the values of the nonlinearity relationships practically coincide. This will undoubtedly increase the correct use of both methods for practical purposes. Furthermore, based on the known value of the intensity effect, the following expression can be written for calculation of the seismic intensity increment:

$$
\Delta /=3 \lg \frac{A_{i} f_{i}}{A_{0} f_{0}}
$$

where $\Delta /$ is the increment of seismic intensity, and $A_{i} f_{i}$ and $A_{0} f_{0}$ are the product of the peak value of the spectrum by the weighted average vibration frequency of the compared soils.

Thus, the formulas are for calculating the increment of seismic intensity based on the degree of nonlinearity of soils; i.e., degrees of deviation from linear-elastic behavior are obtained.

The appearance of constant component of soil field of displacements in the zone of intensive dynamic impacts takes a special place amongst nonlinear effects. Due 444
NPGD

2, 425-474, 2015

Soils' seismic property research on the basis of investigation of their nonlinear properties

V. B. Zaalishvili

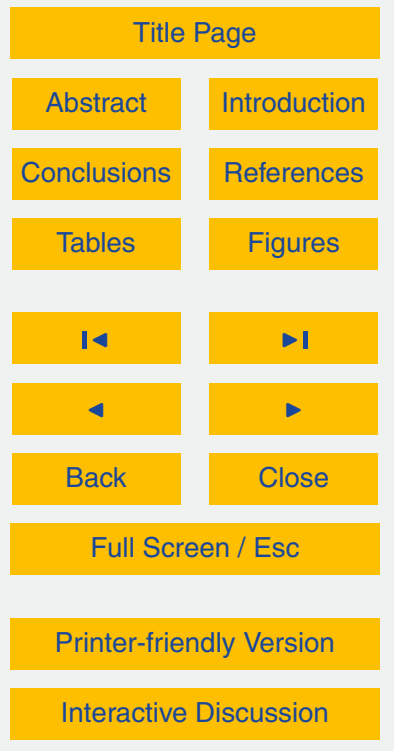

Interactive Discussion 
to the clear nonlinear connection strain deformation at the border of the half-space there exist an inequality of the phases of low pressure and contraction. It is expressed in the origination of the so-called seismic-radiation power, which leads to the typical soil elevation. The investigations showed that at vibration source impact on soil the 5 constant component, which can be measured on the value of stratum surface incline (which decreases with the distance from source, Fig. 7) is formed in the soil.

Impact intensity variation also considerably changes the inclination value (Fig. 8). And, finally, the effect directly depends on soil lithologic compound. Higher values of the angles of inclination are corresponded to weaker soils, i.e. if the pressure change 10 in the vibrator cylinder for more solid (rock) soils leads to the minimum palpable effect, than the effect for the softer soils is quite perceptible. At the maximum pressure of the system of hydrocylinder (180 atmospheres) for sand and asphalt, which is overlying the mentioned sand, the soil low pressures increase quite sharply.

Usage of the value of constant component according to the Fig. 8 has quite clear 15 physical meaning. For example, here the intensity increment of clay soils of relatively weak weathered rock is $2^{\circ}$ according to the formula:

$\Delta /=3 \lg \theta_{i} / \theta_{0}$

where $\theta_{i}$ and $\theta_{0}$ are the inclination angles of the Earth's surface.

In order to apply Eq. (24), it is necessary to use a vibratory energy source. Already 20 the first investigations showed the sufficient reliability of the data and the undoubted prospectivity of the approach. Unfortunately, generalizations of the experimental work with the mentioned parameter were not carried out. Nevertheless, it is possible to recommend it for practical seismic microzonation under the condition of its usage together with the other tools of the instrumental method of SMZ. The offered tool, undoubtedly, 25 will allow one to obtain an important and original index at SMZ, considerably increasing the reliability of the final results.

\section{NPGD}

2, 425-474, 2015

Soils' seismic

property research on

the basis of

investigation of their nonlinear properties

V. B. Zaalishvili

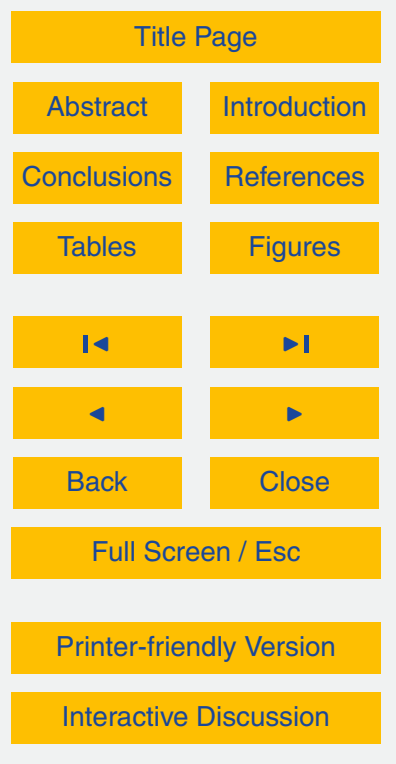




\section{Seismic microzonation based on accounting of inelastic soil properties}

The estimation of potential soil non-elasticity adequately and physically proved at intensive seismic loadings is the most important problem of SMZ as soil liquefaction and differential settlement of the constructions are observed at strong earthquakes (Niigata, 5 1966; Kobe, 1995).

For direct assessment of soil non-elasticity the specific scheme of the realization of experimental investigations (Fig. 9a) with gas-dynamic impulsive source GSK-6M (with two radiators) was used. Chosen location of the longitudinal profile made it possible to impact sequentially by two emitters from near and somewhat far radiation zones. The

component that quickly attenuates with distance (Fig. 9b) prevails in the ground vibrations spectrum, caused by near emitter. In case of distant emitter influence to the ground surface, the LF component prevails in the vibration spectrum (Fig. 9c). Otherwise stated, at nonlinear-elastic deformations, the main energy is concentrated in the $\mathrm{HF}$ range of the spectrum and at non-elastic deformations in the LF range. A symmetrical form is usual for the signal spectrum in the far and practically linear-elastic zone.

For the given source elastic linear and nonlinear vibrations are exemplified by the permanency of the real spectrum square, which is the value index of particular source energy, absorbed by soil (warped by the source). Using the analysis of strong and destructive earthquake records and also the analysis of specially realized experimental impacts it was obtained that at non-elastic phenomena spectra square of corresponding ground vibrations is not the constant value. It may decrease and the more, the less the soil solidity is and the greater the influence value is.

So as to estimate soil seismic hazard accounting the values of their non-elasticity et al., 1996) was obtained using a vibratory energy:

$$
\Delta /=2.4 \lg \left[\left(S_{\mathrm{ri}}\right)_{n}\left(S_{\mathrm{ro}}\right)_{d} /\left(S_{\mathrm{ri}}\right)_{d}\left(S_{\mathrm{ro} 0}\right)_{n}\right]
$$

NPGD

2, 425-474, 2015

Soils' seismic property research on the basis of investigation of their nonlinear properties

V. B. Zaalishvili

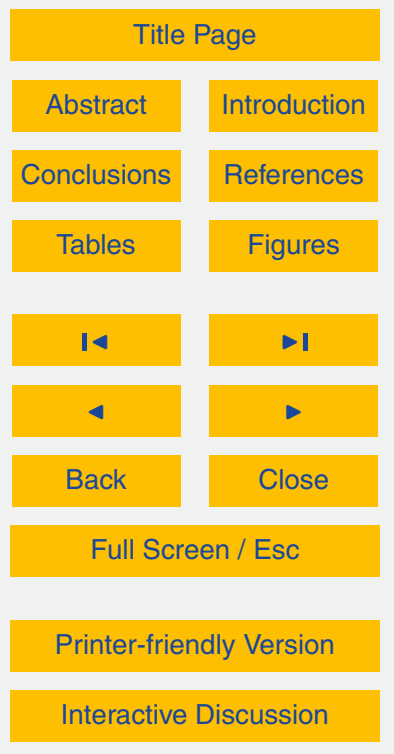


where $\left(S_{\mathrm{ri}}\right)_{n d}$ and $\left(S_{\mathrm{r} 0}\right)_{n d}$ are the squares of real spectra of investigated and reference soils in a source near and distant zones:

$\Delta /=3.3 \lg \left(A_{i} f_{\text {awi }}\right)_{n}\left(A_{0} f_{\text {awo }}\right)_{d} /\left(A_{i} f_{\text {awi }}\right)_{d}\left(A_{0} f_{\text {awo }}\right)_{n}$,

where $\left(A_{i} f_{\text {awi }}\right)_{n d}$ and $\left(A_{0} f_{\text {awo }}\right)_{n d}$ are the amplitudes and weighted average frequencies 5

of the investigated and reference soils in a source nearby and distant zones.

If a powerful impulsive source is using the afore-cited formulas, they will have a form

$\Delta /=1.2\left[\lg \left(S_{\mathrm{ri}}\right)_{n}\left(S_{\mathrm{r} 0}\right)_{d} /\left(S_{\mathrm{ri}}\right)_{d}\left(S_{\mathrm{r} 0}\right)_{n}\right]$,

where $\left(S_{P_{i}}\right)_{n d}$ and $\left(S_{P 0}\right)_{n d}$ are the squares of real spectra of investigated and reference soils in a source near and distant zones:

$\Delta /=2 \lg \left[\left(A_{i} f_{\mathrm{aw} i}\right)_{n}\left(A_{0} f_{\mathrm{aw} 0}\right)_{d} /\left(A_{i} f_{\mathrm{aw} i}\right)_{d}\left(A_{0} f_{\mathrm{aw} 0}\right)_{n}\right]$,

where $\left(A_{i} f_{\text {awi }}\right)_{n d}$ is the amplitude and $\left(A_{0} f_{\text {aw }} 0\right)_{n d}$ is weighted average frequency of investigated and reference soils in a source nearby and distant zones.

Eqs. (25) and (27) are adequate only for soft-dispersed soils. Eqs. (25) and (26) were used at SMZ of the Kutaisi city territory. Moreover, using Eqs. (27) and (28) (Zaalishvili

and Timoshuk, 1996; Zaalishvili et al., 1996), non-elastic deformation soil properties under full-scale conditions on the Novovoronezh APP-2 site were defined more accurately. The formulas were obtained based on the physical principle that underlies the scheme used at the soil looseness assessment (Nikolaev, 1967; Zaalishvili, 1996).

Modern powerful vibratory seismic sources (SV-10/100, VSH-8 and others) are acceptable as vibration sources at usage of Eqs. (25) and (26) and at usage of Eqs. (27) and (28) the impulsive sources (SI-32, GSK-6, GSK-10 and others) are suitable (Shneerson, Mayorov, 1980).

\section{Seismic microzonation in the case of variable seismic impact level}

Damage of the system soil construction during seismic impact occurred due to system inability of absorbing the corresponding seismic energy. After a partial damage the sys-
NPGD

2, 425-474, 2015

Soils' seismic

property research on

the basis of

investigation of their nonlinear properties

V. B. Zaalishvili

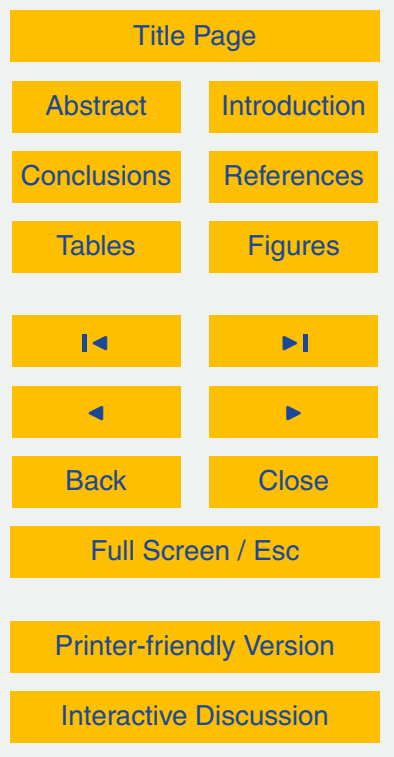


tem gets additional intake, which preserves it from the future damages. At continuing introduction of the energy, the system will be more damaged, thereby increasing its intake until the seismic impact does not stop or the damage level becomes incompatible with its bearing element integrity, and the system self-destructs. In this connection, 5 very important problem is to assess adequately seismic absorption of energy by physical systems of different types or by their combinations (soils, buildings, constructions, etc.), which take part in forming of strong earthquake intensity. Theoretical solutions of the given problem often considerably differ from the real results. It causes great practical and scientific interest to the results of analysis of the instrumental records of strong 10

\subsection{Soft and firm soils}

What is the difference between soft and firm soils? For the first time the interconnection of a number of different indices of ground vibrations was investigated by the author on the data of station at Racha earthquake in 1991. It was determined that simple quantitative measure of spectrum energy absorption is the square of normalized vibration spectrum (Zaalishvili, 2000, 2001).

The square of normalized vibration spectrum for the firm soils (station Oni) increases and in the firm soils (station Iri) the square decreases with vibration acceleration (Fig. 2a). Maximum amplitudes of vibration spectra of soft and firm soils are in direct 20 proportion to vibration acceleration (Fig. 2b).

Vibration acceleration as the index of influence is widely used in different investigations in engineering seismology and earthquake engineering. However, its often bad correlation with earthquake magnitude, which is in inverse ratio to the ground vibration frequency, is well known. As a consequence of analysis, it was determined that the 25 ratios, where the weight-average value of vibration frequency is used instead of maximum values of vibration frequencies, are characterized by considerably less variety in the correlated parameters (Zaalishvili, 1986).
NPGD

2, 425-474, 2015

Soils' seismic property research on the basis of investigation of their nonlinear properties

V. B. Zaalishvili

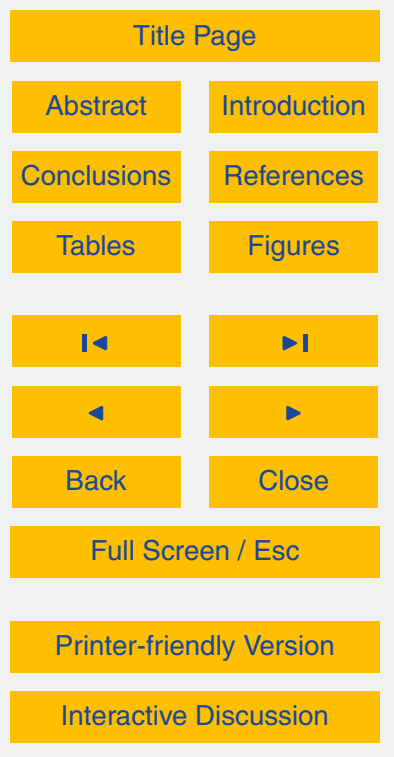


The dependence of real vibration spectra squares of soft soils (a) and rocks (b) on acceleration is shown in the Fig. 10. One can see that the square or real vibration spectrum for rocks increases with the growth of vibration frequency, and for soft soils the square decreases (Fig. 10a). It is interesting to compare the mentioned dependences, 5 which describe the behavior of viscoelastic material, where a real part of the solution of motion equation corresponds to an insignificant rigidity decrease with the frequency (Fig. 10b). Good correspondence of experimental and theoretical data was determined at their comparison.

So, first of all, the square of a real vibration spectrum is a reliable indicator of the 10 physical condition of the medium and, in the second place, it describes the medium deformability or its behavior deviation scope from the linear elastic law of Hooke.

Thus, the square of the real vibration spectrum is an important index of soil nonlinear behavior at different impact levels.

In this connection, it must be noted that, for the SMART 1 system (Sect. 2), consid15 erable nonlinearity is displayed from the acceleration $\mathrm{a}=0.1 \mathrm{~g}$ (Fig. 11). Similarly, at the acceleration $a=0.1 \mathrm{~g}$, the "break" in the dependence curve of the square of the real spectrum on acceleration for station Zemobari begins (Fig. 4b).

\subsection{Weak and strong soil motions}

What does the strong motion mean and how does it differ from the weak motion? 20 At strong motion, clear reconstruction of the physical system begins to this or that extent. From the point of view of earthquakes, the influence of nonlinear phenomena on seismic effect, i.e., earthquake intensity, is clearly displayed at strong motion. In 1996, we experimentally investigated in the area, composed of powerful sediments, near the city of Noviy Voronezh, how the ground vibration spectrum depends on impact level 25 (Zaalishvili, 2001, 2009). A powerful gas-dynamic non-explosive source GSK-6M with two emitters of impulse vibrations was used as the vibration source.

The analysis of vibrations, initiated by near emitter, shows that the main energy in the near zone of the source is contained in the range HF field, which quickly attenuates

\section{NPGD}

2, 425-474, 2015

Soils' seismic property research on the basis of investigation of their nonlinear properties

V. B. Zaalishvili

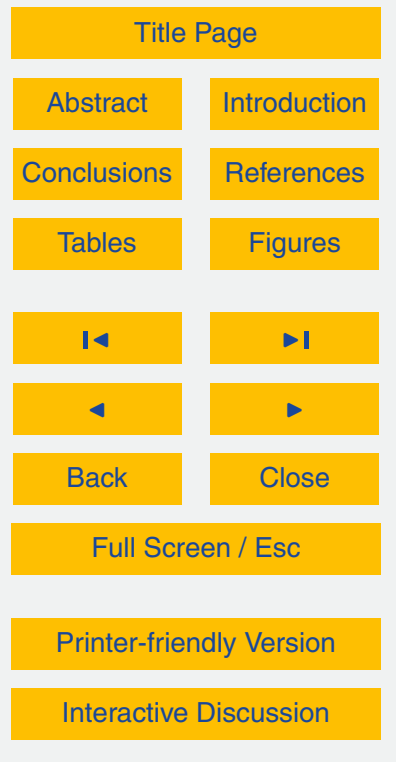


with distance (Fig. 9). At distant emitter the LF field prevails and HF component, which attenuates rapidly on record, is insignificant.

Thus, it is interesting to compare the findings and the results of calculations of French scientists (Bonnet, Heitz, 1994) (Fig. 12). One can clearly see that at the transformation

5 of linear-elastic into nonlinear-elastic deformations, the energy remains constant and "transfers" to the HF spectrum range, and the square of the real vibration spectrum does not change.

Nonlinear extension of wave shapes, occurring in the so-called "bimodule" medium (Nikolaev, 1967; Zaalishvili, 2009), is characterized by the spectrum "transferring" into 10 the region of low and high frequencies. "Bimodularity" is the medium property, which is characterized by the difference in its deformation modules of contraction and extension.

At that one and the same medium at contraction can have one value of the module and at extension may have another value. These mediums are real soils, the modules of contraction and extension have difference, which are larger when the level of defor15 mations is greater and the soil is worse on its seismic properties. As an example of extreme difference of modules value we can consider the stratum, composed with dry sand, which has heavy module at contraction and zero module at extension.

\subsection{Seismic impact and soil response}

It was earlier obtained by us that the product of the maximum amplitude of the vibration 20 spectrum and the weight-average frequency of vibrations is the simplest and easiest way of measuring the index, which describes nonlinearity - a special property of the medium.

Let us try to obtain the same result in some other way. With the help of correlation analysis of the data (records of Racha earthquake, 1991), which were obtained using $25 \mathrm{SMACH}$, it was determined that vibration absorption in soft soils within $10-40 \mathrm{~km}$ from the source (aftershocks of the earthquakes with magnitude $M=1.2-6.3$ ) is proportional to the expression (Zaalishvili, 2000)

\section{NPGD}

2, 425-474, 2015

Soils' seismic property research on the basis of investigation of their nonlinear properties

V. B. Zaalishvili

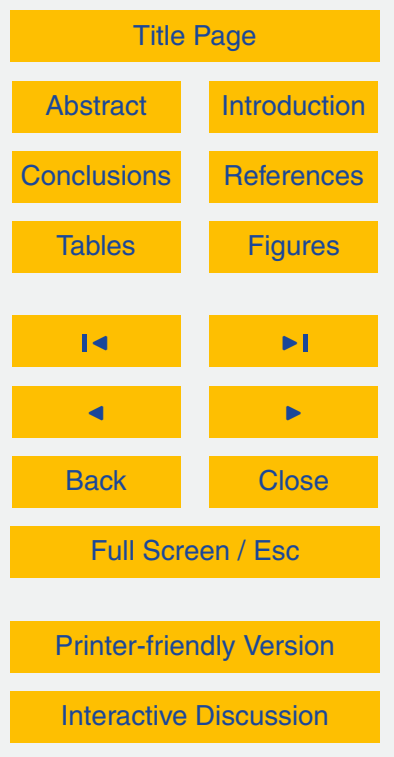


where $t$ is the vibration duration, and $M$ is the earthquake magnitude.

In rocks or firm soils,

$\alpha \sim f^{2} t \sqrt{a r}$

5 where $a$ is the peak of soil acceleration, and $r$ is the epicenter distance.

So, absorption of vibration practically does not depend on acceleration in soft soils. Magnitude fully determines its level: the larger amplitude the less absorption. Absorption is in direct proportion with soil acceleration in rocks. Such "dualism" of seismic impact means that at calculation of behavior of buildings and constructions, which are 10 raised on soft soils, it is necessary to realize energy calculation, i.e., by usage of energy introduction of definite reference energy and for buildings, which are raised on rocks accelerations.

It is important to use energy characteristics of object motions at strong seismic and, obviously, dynamic impacts. The results of macroseismic observations of strong and destructive earthquake consequences point to that fact. On the other hand at comparison of vibration parameter in the form of the square of real spectrum $S_{\mathrm{R}}$ for the corresponding soils from soft soil of Eqs. (10), (14) and (28) and taking into account that $A \sim M^{2.5} \sqrt{a}$ (Zaalishvili, 2000), we have the ratio

$\Delta I=K \lg \frac{f_{\mathrm{w} i}^{2} M_{i}^{2} t_{i}}{f_{\mathrm{w} 0}^{2} M_{0}^{2} t_{0}} \sqrt{\frac{a_{i}}{a_{0}}}$,

20 where $f_{\text {wio }}$ is the weight-average vibration frequency at different magnitudes, respectively; $M_{i 0}$ are magnitudes of different impacts, accordingly; and $a_{i 0}$ is the value of soil acceleration at different magnitudes, accordingly.

Soils' seismic property research on the basis of investigation of their nonlinear properties

V. B. Zaalishvili

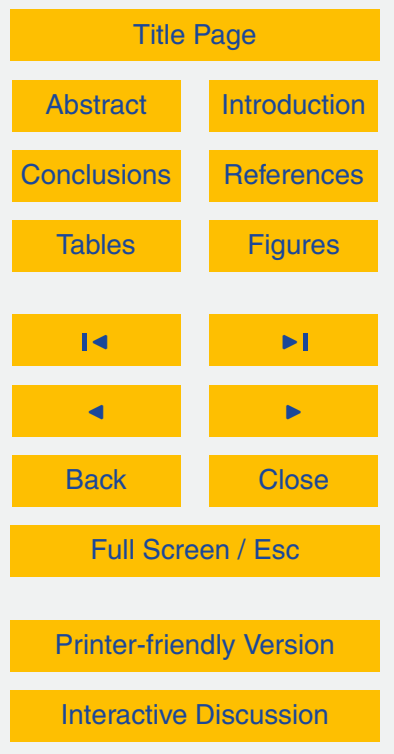


Eq. (30) allows one to assess the influence of impact energy change (magnitude) on the value of the intensity increment on the given soil. In other words, we obtained the expression for the direct calculation of a nonlinear increase of the given soil.

Furthermore, the expression is obtained that allows one to determine how energy 5 impact influences the intensity increment for soft soils, comprising different areas of the territory (Zaalishvili, 2004):

$\delta I=2\left\{\lg \frac{M_{1}^{2} t_{i 1} f_{\mathrm{w} i 1}^{2}}{M_{2}^{2} t_{i 2} f_{\mathrm{w} i 2}^{2}} \sqrt{\frac{a_{i 1}}{a_{i 2}}}-\lg \frac{M_{1}^{2} t_{01} f_{\mathrm{w} 01}^{2}}{M_{2}^{2} t_{02} f_{\mathrm{w} 02}^{2}} \sqrt{\frac{a_{01}}{a_{02}}}\right\}$,

where $\delta I$ is nonlinear increase at variable impact level, $\delta I=\Delta I_{n i}-\Delta I_{n 0} ; \Delta I_{n i}, \Delta I_{n 0}$ is nonlinear increase for investigated and reference soils, accordingly, degree; $t_{i 01}$ and

${ }_{10} t_{i 02}$ are the duration of vibrations of investigated and reference soils at $(n)$ and $(n+1)$ earthquakes (with magnitudes $M_{1}$ and $M_{2}$ ), accordingly, s; $f_{\text {wi01 }}$ and $f_{\text {wi02 }}$ are weighted vibration frequencies of soil under investigation and reference soil at $(n)$ and $(n+1)$ earthquakes, accordingly, $\mathrm{Hz}$; and $a_{i 01}$ and $a_{i 02}$ are vibration accelerations of investigated and reference soils at $(n)$ and $(n+1)$ earthquakes, accordingly, $\mathrm{ms}^{-2}$.

15 Let us consider the example. The results of the comparison of the engineering macroseismic investigation in the Racha earthquake epicentral zone (Georgia, 1991) and the parameters of instrumental records, which were obtained by the SMACH network, under different soil conditions, are given in Table 1 . The calculations of intensity increase were carried out by means of the above-considered ratio.

One can see well that, with earthquake magnitude increase, the intensity increment decreases. It explains in many respects the considerable difference in the features of soil vibrations in near and far zones. So, the small difference in seismic effects even between the soils with quite various seismic properties is well known. The nonlinear stress-strain relationship of soft soil causes unalike distortion of the phases of compression and stretching and the increase in the phase of low pressure in the softer soils, which leads to the dependence of dynamic indices of soil motion on the impact energy.

\section{NPGD}

2, 425-474, 2015

Soils' seismic

property research on

the basis of

investigation of their nonlinear properties

V. B. Zaalishvili

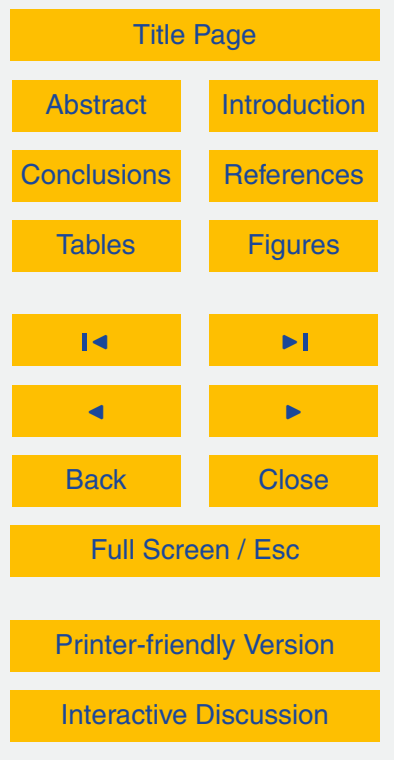


But, let us return to Eq. (31). Let $M \sim E$ (artificial source energy). Taking into account that, for artificial vibrator $M_{i} \approx M_{0}, t_{i} \approx t_{0}$, and $a \approx A_{2}$, we got the expression, which is similar to Eq. (15),

$\Delta /=K \lg \left(A_{i} f_{\mathrm{w} i} / A_{0} f_{\mathrm{wo}}\right)$.

5 Taking into account the impact level on calculation of the intensity increment, we actually take into account soil nonlinearity. At that, the formula for intensity increment computation based on the comparison exceptionally of indices of the corresponding soil nonlinearity degree can be obtained in terms of the connection between the parameters of seismic impact and characteristics of soil conditions, i.e., in another way. It undoubtedly increases the foundation of its usage in practical works of seismic microzonation.

It is interesting that, at small differences of soil weight-average frequencies, i.e., when $f_{1} \approx f_{2}$, Eq. (32) is transformed to the known formula of $\mathrm{S}$. V. Medvedev (Medvedev, 1962):

${ }_{15} \Delta /=K \lg \left(A_{1} / A_{2}\right)$,

where $A_{1}$ and $A_{2}$ are vibrational amplitudes of comparing soils.

Thus, the indices of nonlinearity differ from linear vision exceptionally by the presence or taking into account of vibration frequency values in the corresponding expression. This question needs more strict investigation, as the existing tools of SMZ, which are empirical, take into account soil nonlinearity to this or that extent. But, nonlinearity at that is taken into account in the best case; likewise linearized solutions of nonlinear equations, which are strongly not up to their accuracy. And even if, in some cases, the results approximate to real displays of nonlinearity, then they, firstly, can make larger mistakes and, secondly, they do not need testing. Thus, the problem consists in taking soil nonlinear properties into account in a stricter way at the assessment of soil seismic properties.
NPGD

2, 425-474, 2015

Soils' seismic property research on the basis of investigation of their nonlinear properties

V. B. Zaalishvili

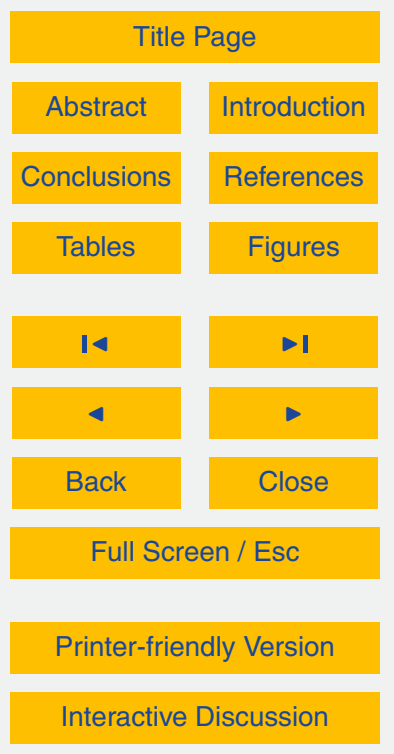




\subsection{Soil patterns of behavior at variable impact intensity}

Aforesaid shows how important it is to investigate soil nonlinear indices in order to assess their seismic properties. In particular, the parameter of the "real spectrum square" is necessary for soil intensity increment calculation, accounting for their nonlinearity 5 degree. In this connection, it is interesting from scientific and practical points of view to consider how values of real spectrum squares depend on impact intensity. In other words, it is important to investigate soil behavior at variable impacts.

At intensive dynamic loads in "weak" (and sometimes not weak) soils or soils highly sensitive to the external impact, the conversion from one mode of deflection to another, 10 particularly, conversion from elastic conditions to non-elastic, takes place. It considerably changes the soil condition influence on the visible intensity effect of an earthquake, which is characterized by the value of a real vibration spectrum square.

It was shown previously that the non-elasticity phenomenon is characterized by the decrease in values of a real spectrum square. Such a decrease is caused by the reduction, in turn, in elastic absorption of energy introduced by the soil stratum. Indeed, at increasing influence within elastic deformations, energy absorption grows in direct proportion to intensity. If the given soil (a real soil stratum with a definite lithologic compound of a pack of soil strata) reaches an ultimate stress or if the soil exhausts the potentialities of energy intensity absorption, then soil structural links get broken. Non-elastic deformations of soil appear at that. It causes a decrease in the energy, consumable on the formation of proper vibration, i.e., elastic motion of particles at the position of their balance. In connection with the fact that a real spectrum square is in direct proportion to the energy absorption, the corresponding decrease in a real spectrum square should be expected.

25 The sharpest absorption decrease corresponds to nonlinear process, when the signal frequency moves to the HF spectrum region, which is highly absorbed by soils. Spectrum "exhaustion" of HF components slows down an absorption velocity. At large intensities, the spectrum peak value (maximum amplitude), which leads to the increase
NPGD

2, 425-474, 2015

Soils' seismic

property research on

the basis of

investigation of their nonlinear properties

V. B. Zaalishvili

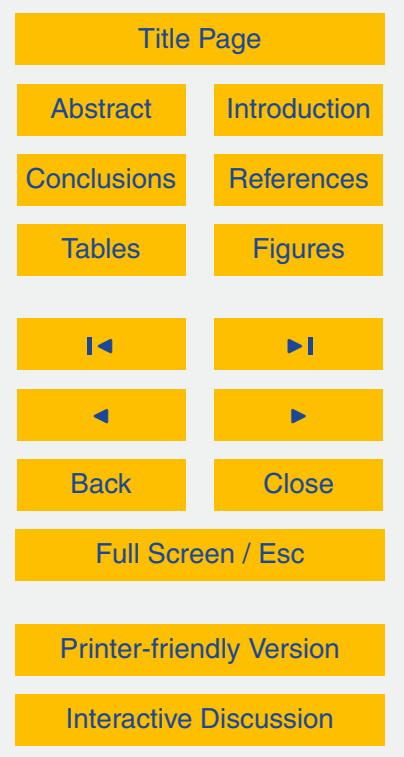


in absorption by "newly formed" soil mass, etc., begins to play the main role. The absorption will not increase during loading in the case of the soil liquefaction, but not soil "shaking up", which brings consolidation to it.

Thus, the real spectrum square that results, integral characteristics of simultaneous 5 influence of the phenomena of absorption, dispersion and nonlinearity, will react to the indefinitely small displays of soil behavior features at intensive variable impact.

Let us consider different models of stress-strain behavior of soils at variable levels of seismic impact (Zaalishvili, 2000) (Fig. 13).

At the relatively small impact, soil strata are characterized by a linear-elastic pattern 10 soils relative to the frequency of rocks, the motion of which can be considered as a real spectrum square.

At the further impact increase, the moment when some part of the vibration energy 15 "transfers" to the HF spectrum region, begins. The square of the real spectrum at that does not change. The peak value decreases with a simultaneous frequency shift. This is the so-called nonlinear-elastic soil pattern of behavior. The following increase in the impact intensity brings the decrease in the real spectrum square. The soil is characterized by nonlinear-elastic behavior (frequency moves to the LF spectrum region). And, finally, at quite strong load frequency shift to the HF field is absent; it is directed to the LF spectrum region. The real spectrum square remains small or decreases even further. This is the so-called linear-non-elastic soil deformation.

The features of vibration spectra of soil stratum, composed of clay in Leninakan (Guymri nowadays) and in Kirovakan, can be considered to be the example (Fig. 14, Khalturin et al., 1989). Such factual data, undoubtedly, must be subjected to a deeper analysis than is realized at present.

Aforesaid shows that, on the one hand, the intensity increment, which is calculated, for example, on the basis of a value of real vibration spectrum squares, has a physical meaning exceptionally within linear-nonlinear elastic deformations. On the other hand,
NPGD

2, 425-474, 2015

Soils' seismic

property research on

the basis of

investigation of their nonlinear properties

V. B. Zaalishvili

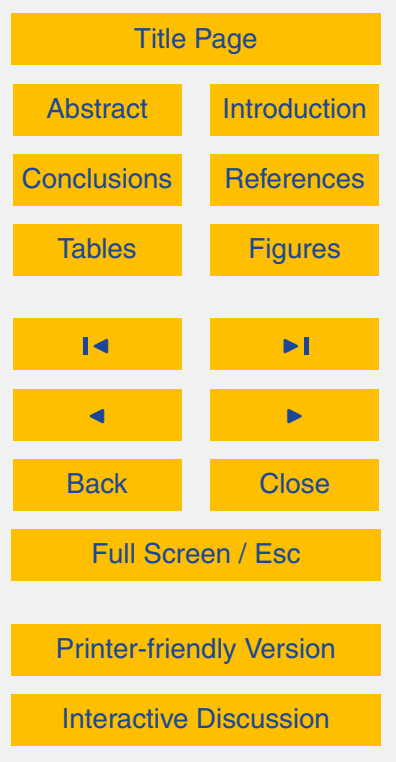


the obtaining of a final, non-zero value of the increment for soils, which are logically characterized by it, is the inherent index of a definite physical condition of elastic soil.

\section{Conclusions}

All empirical tools of SMZ suppose (or they must suppose) the taking into account of 5 nonlinear soil properties. It lies in the SMZ essence. At the same time, the problem, obviously, consists in the following: how soil nonlinearity is taken into account and how impartial such a taking into account is.

The execution of the practical realization of the techniques of soil nonlinearity determination on the basis of assessment of their strain sensitivity (vibro-sensitivity), con10 stant component of displacement and square of real vibration spectrum in areas with known displayed intensity of destructive earthquakes allowed one to obtain a number of proven conclusions.

The identity of the nonlinearity index was determined in the form of strain-sensitivity characteristics and introduced again the index in the form of the product of the value of 15 the peak spectrum on the weighted frequency of ground vibrations of compared areas. The empirical formula allows one to calculate the intensity increment by taking "pure" nonlinearity of soils into account.

Execution of calculation analysis on impulse and vibration impact allowed one to validate the techniques of the assessment of soil nonlinearity on the basis of comparison results of numerical and experimental methods. The techniques of the assessment of the soil nonlinearity degree on the basis of comparison of numerical methods in linear settings and real vibration spectra are given.

The parameters, closely connected with absorption and soil nonlinearity, were disclosed on the basis of the analysis of strong motion instrumental records. The empirical formulas, which connect the squares of normalized and real spectra with the parameters of seismic impact (magnitude, acceleration, epicentral distance, duration and weight-average frequency of ground vibrations), are obtained by regression anal-
NPGD

2, 425-474, 2015

Soils' seismic property research on the basis of investigation of their nonlinear properties

V. B. Zaalishvili

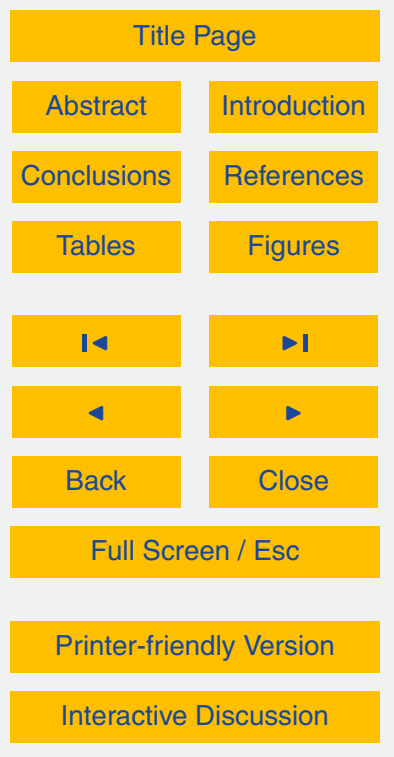


ysis. The difference between absorption mechanisms in dispersal (soft) soils and rocks was determined. The proportionality of the soil nonlinearity degree to the product of the amplitude of a vibration spectrum peak value at the weight-average frequency was confirmed on the basis of the conception of the integral influence of the phenomena of 5 absorption and nonlinearity on the resulting soil motion. Taking into account the impact level allows one to avoid usage of the traditional (obviously incompatible with nature) constancy of intensity increment.

The absorption is mainly determined by earthquake amplitude in soft soils, while in firm soil, it is determined by acceleration. In the near zone of the source at non10 linear deformations, the energy extends to the high-frequency range of spectrum and the phenomena of considerable absorption are observed. Sometimes it causes the mixture of "absorption" and "nonlinearity" phenomena. The spectrum is saturated by high-frequency components at nonlinearity display and, at the absorption, the spectrum becomes low frequency.

15 For registration of ultra-high accelerations (at nonlinear deformations in the epicentral zone of the earthquake), it is necessary to extend the level of registration of soil accelerations upward from the traditionally accepted boundary of $2 \mathrm{~g}$. It is necessary to improve completely the level of data resolution at analysis of hard motion in order to disclose momentary or "short-lived" ultra-high reactions.

Soil patterns of behavior (at dynamic impact of variable intensity) are offered on the basis of the consideration of a real spectrum square and a value of the weight-average frequency, which characterize linear-nonlinear and elastic-non-elastic soil deformation.

Acknowledgements. I would like to express my deepest appreciation to all those who provided me with the possibility of completeing this report. A special gratitude I give to my colleagues PhD D. Melkov, PhD O. Burdzieva and PhD B. Dzeranov. Furthermore I would also like to acknowledge with much appreciation the crucial role of Federal Targeted Program "Scientific and Scientific-and-pedagogic Community of Innovative Russia" 2009-2013, state contract \#02.740.110733 dated 5 April 2010 for financial support in conducting investigations.

\section{NPGD}

2, 425-474, 2015

Soils' seismic property research on the basis of investigation of their nonlinear properties

V. B. Zaalishvili

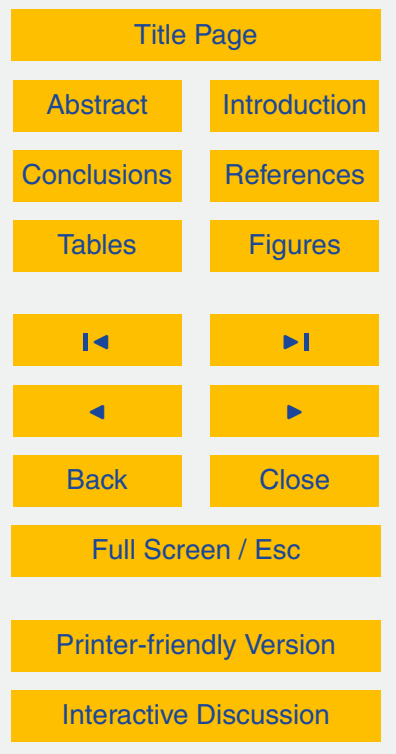




\section{References}

Beresnev, I. A., Solovyev, V. S., and Shalashov, G. M.: Nonlinear and parametric phenomena in seismicity of harmonic vibration signals, in: Problems of Nonlinear Seismology, Nauka, Moscow, 180-185, 1987.

5 Dzhindzholava, B. S.: Evaluation of the sensitivity of metrological parameters of measuring and computing systems in operation modes, in: Methods and Means of Metrological Assurance: Coll. Works NMI, Metsniereba, Tbilisi, 72-75, 1986.

Engelbrecht, Y. K. and Feldman, M. V.: Change in the spectral composition of the seismic pulses during propagation in a nonlinear medium, in: Problems of Nonlinear Seismology,

10 Nauka, Moscow, 103-108, 1987.

Khalturin, V. I., Rautian, T. G., Sargsyan, N. M., and Shomahmadov, A. M.: Strengthening microseismic effect in Leninakan, in: Synthesis and Evaluation of the Preliminary Results of the Investigations and Survey of Earthquakes, Iss. of Acad. Sci. of Armenia, Yerevan, 11-13, 1989.

15 Lehmann, E. L.: Testing of Statistical Hypotheses, Nauka, Moscow, 498 pp., 1964.

Mandelshtam, L. I.: Complete Set of Works. V, III. Publishing House of the Academy of Sciences of the USSR, Moscow, 421 pp., 1950.

Medvedev, S. V.: Engineering Seismology, Gosstroiizdat, Moscow, 284 pp., 1962.

Nikolaev, A. V.: Seismic properties of loose medium, in: Izvestia, Ser. Physics of the Earth, USSR Academy of Sciences, Moscow, 23-31, 1967.

Nikolaev, A. V.: Problems of nonlinear seismology, in: Problems of Nonlinear Seismology, Nauka, Moscow, 5-20, 1987.

Schneerson, M. B. and Mayorov, V. V.: Land Seismic Sources With Non-Explosive Sources of Vibrations, Nedra, Moscow, 205 pp., 1980.

25 Trifunac, M. D.: Response spectra of strong motion acceleration: extension to high and low frequencies, in: Proc. 10th European Conf. on Earthquake Eng., vol. 1., Vienna, 1994.

Vasiliev, Y. I., Ivanova, L. A., and Scherbo, M. N.: Measurement of stresses and strains in the soil during the propagation of blast waves, in: Izvestia, Ser. Physics of the Earth, USSR Academy of Sciences, Moscow, 21-37, 1969.

so Vasiliev, Y. I., Vidmont, N. A., Gvozdev, A. A., Sokolov, V. L., Khavroshkin, O. B., and Scherbo, M. N.: Direct measurements of seismic radiating stress in the soft ground, in: Problems of Nonlinear Seismology, Nauka, Moscow, 149-152, 1987.

\section{NPGD}

2, 425-474, 2015

Soils' seismic

property research on

the basis of

investigation of their nonlinear properties

V. B. Zaalishvili

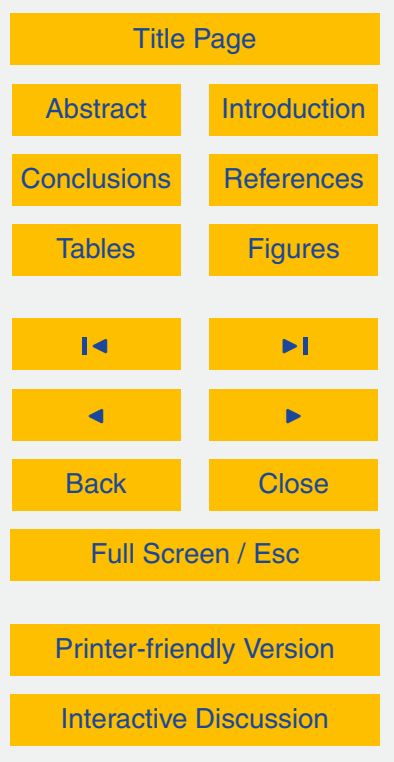


Zaalishvili, V. B.: Seismic Microzonation According to Artificial Initiation of Vibrations of a Ground Layer, Author. Dis. Sciences Candidate, Institute of Geophysics of Georgian Academy of Sciences, Tbilisi, 1986.

Zaalishvili, V. B.: Weighted average period of ground motion initiated by various kinds of artificial sources, in: Proc. Conf. Joint session of the Research Institute for the construction of the Transcaucasian Republics, Metsniereba, Tbilisi, 70-71, 1987.

Zaalishvili, V. B.: Seismic Microzonation Based on the Investigation of Nonlinear Properties of Soils Artificial Sources, Author. Dis. Sciences Doctor, MSU, Moscow, 1996.

Zaalishvili, V. B.: Physical basis of seismic microzonation, UIPE RAS, Moscow, 367 pp., 2000.

Zaalishvili, V. B.: Strong motion in absorbing nonlinear medium and problems of their registration, in: Strong Motion Instrumentation for Civil Engineering Structures, Kluwer Academic Publishers, Istanbul, 611-621, 2001.

Zaalishvili, V. B.: Seismic microzonation with a varying level of seismic impact, in: Proceedings of V All-Ukrainian Conference on Earthquake Engineering, Yalta, NDIBK, Budivelni konstruktsii konstruktsii. Iss. 60, Kiev, 149-156, 2004.

Zaalishvili, V. B.: Seismic Microzonation of Urban Territories, Towns and Large Construction Sites, Nauka, Moscow, 350 pp., 2009.

Zaalishvili, V. B.: Assessment of seismic hazard of territory, in: Earthquake Engineering, edited by: Sezen, H., InTech, Rijeka, doi:10.5772/48324, 2012.

20 Zaalishvili, V. B. and Timoshuk, E. P.: Seismic Microzonation of 3-4th Blocks Site of Novovoronezh NPP-1 Using Microseisms, Report AO ISGIM, Institute Funds "AEP", Moscow, 1996.

Zaalishvili, V. B., Timoshuk, E. P., Kuranova, O. I., and Labzina, E. V.: Specification of Inelastic Deformation Properties of Soils Using Seismic Methods in Natural Conditions at the Site 1996.

\section{NPGD}

2, 425-474, 2015

Soils' seismic

property research on

the basis of

investigation of their nonlinear properties

V. B. Zaalishvili

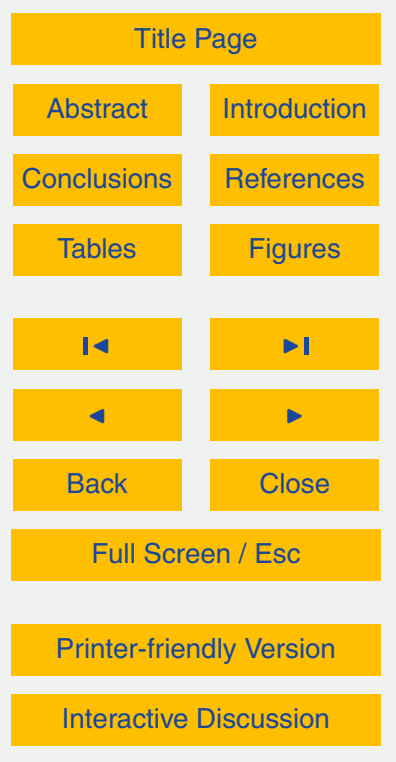


Table 1. Engineering-geological conditions and parameters of the corresponding SMACH network instrumental records.

\begin{tabular}{lccc}
\hline $\begin{array}{l}\text { Engineering-geological conditions } \\
\text { of the site }\end{array}$ & $\begin{array}{l}\text { Intensity increment, } \Delta / \text {, degree } \\
\text { at earthquake magnitude }\end{array}$ \\
\cline { 2 - 4 } & $M=3.0$ & $M=5.0$ & $M=5.3$ \\
\hline $\begin{array}{l}\text { (a) Macroporous clay }(h=10.0 \mathrm{~m}) \\
\text { (b) Pebbles with sandy argillaceous filler }\end{array}$ & - & - & - \\
$\begin{array}{l}(>30 \%, h=5.0 \mathrm{~m}) \\
\text { (c) Weakly weathered limestones }\end{array}$ & & & \\
\hline $\begin{array}{l}\text { (a) Weathered limestones }(h=10.0 \mathrm{~m}) \\
\text { (b) Weakly weathered limestones }\end{array}$ & -2.30 & -1.48 & -0.84 \\
\hline
\end{tabular}

NPGD

2, 425-474, 2015

Soils' seismic property research on the basis of investigation of their nonlinear properties

V. B. Zaalishvili

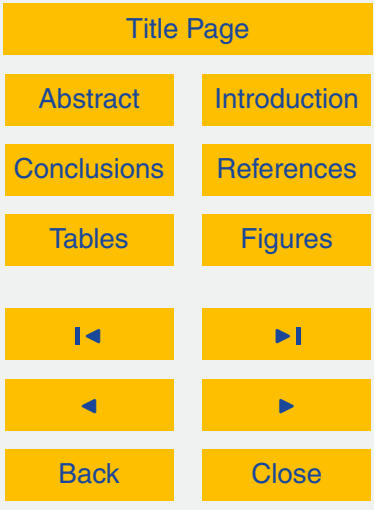

Full Screen / Esc

Printer-friendly Version

Interactive Discussion 


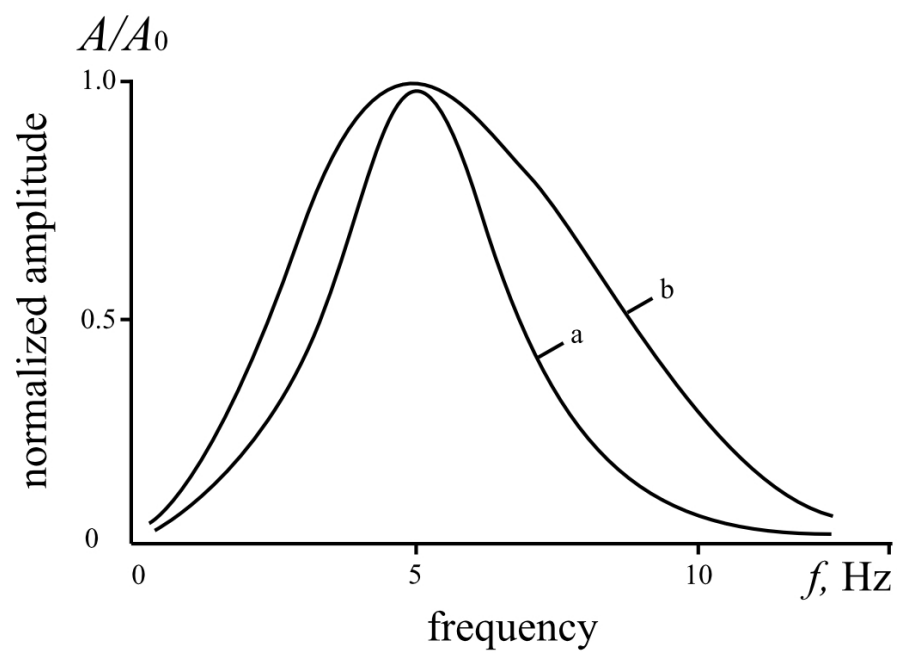

Figure 1. Evolution of the initial seismic signal (a) in the nonlinear elastic medium (b).

\section{NPGD}

2, 425-474, 2015

Soils' seismic property research on the basis of investigation of their nonlinear properties

V. B. Zaalishvili

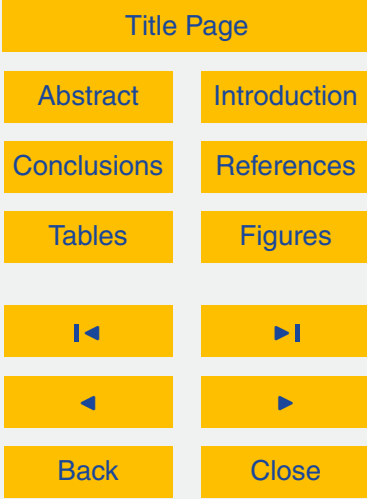

Full Screen / Esc

Printer-friendly Version

Interactive Discussion 

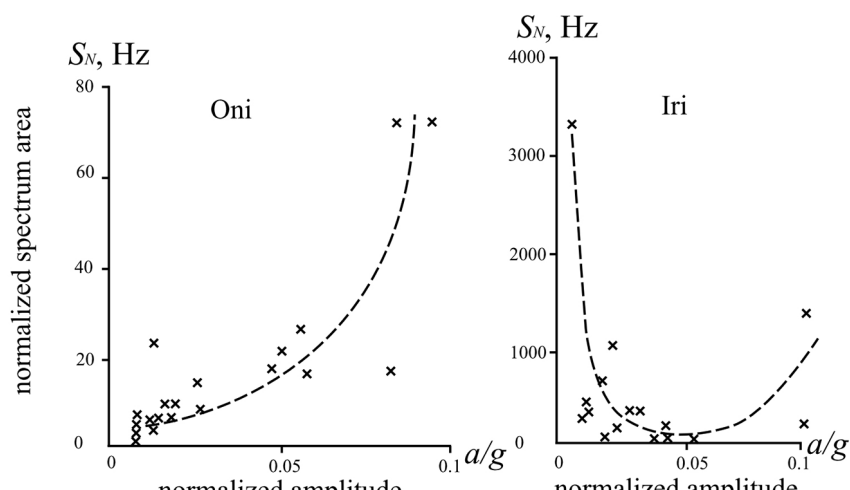

normalized amplitude normalized amplitude

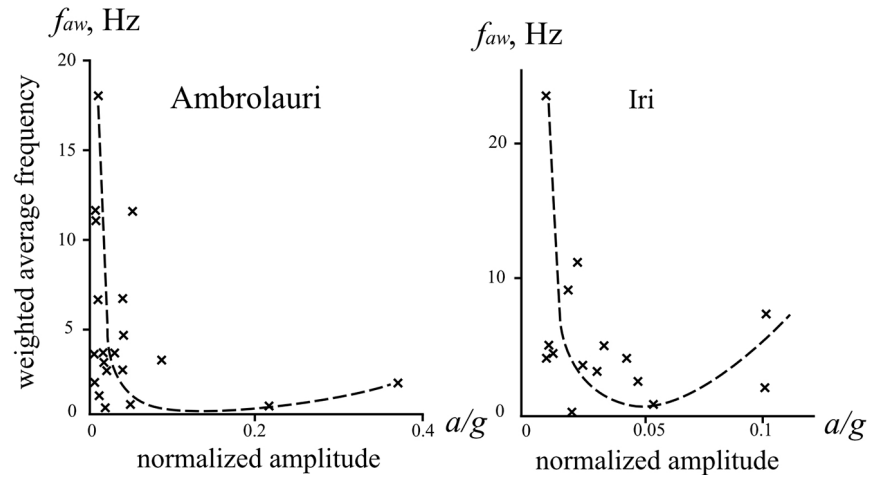

Figure 2. Indices of soil motion in the function of normalized acceleration ag ${ }^{-1}$ (Georgia, 1991).
NPGD

2, 425-474, 2015

Soils' seismic property research on the basis of investigation of their nonlinear properties

V. B. Zaalishvili

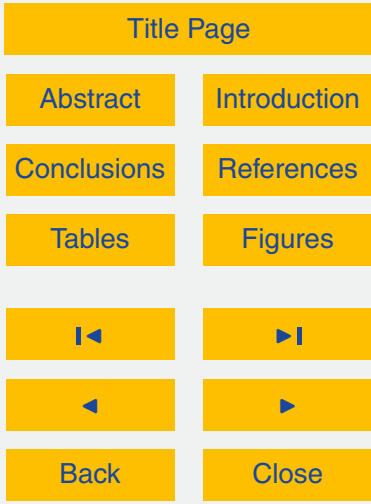

Full Screen / Esc

Printer-friendly Version

Interactive Discussion 


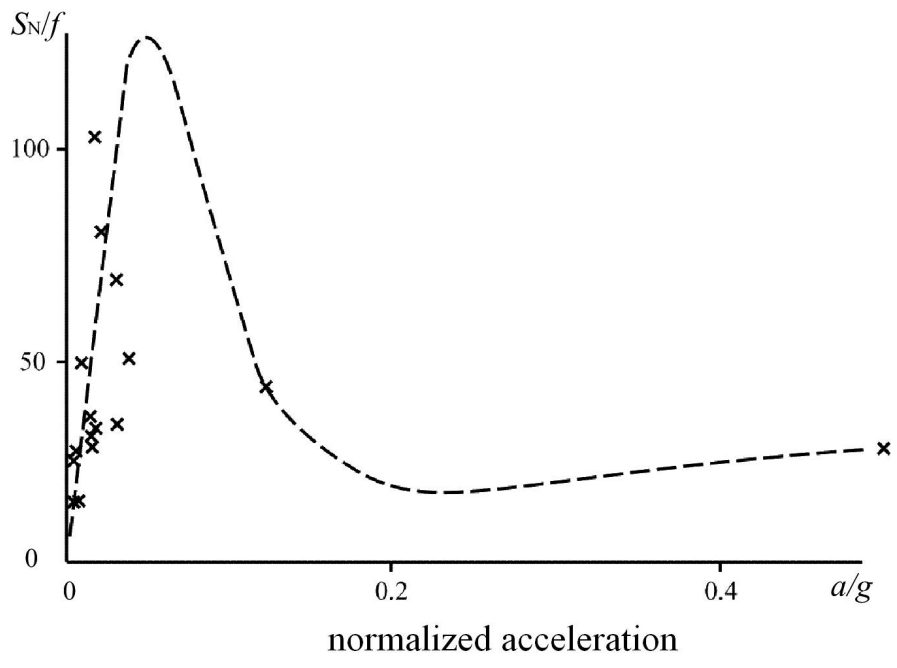

Figure 3. Dependence of the $S_{\mathrm{N}} / f_{\text {aw }}$ parameter on normalized acceleration $\mathrm{ag}^{-1}$.

\section{NPGD}

2, 425-474, 2015

Soils' seismic property research on the basis of investigation of their nonlinear properties

V. B. Zaalishvili

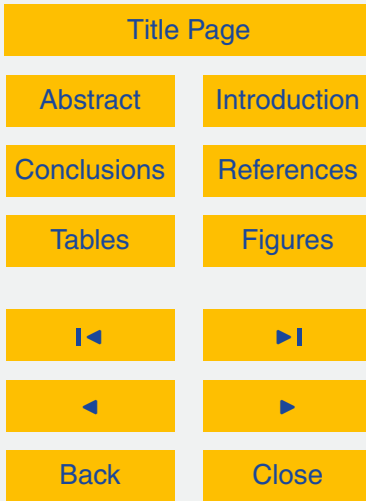

Full Screen / Esc

Printer-friendly Version

Interactive Discussion 


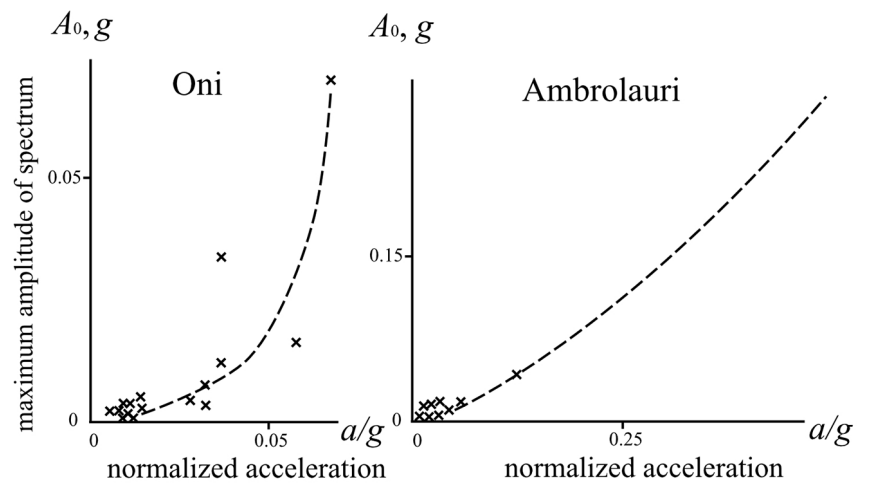

NPGD

2, 425-474, 2015

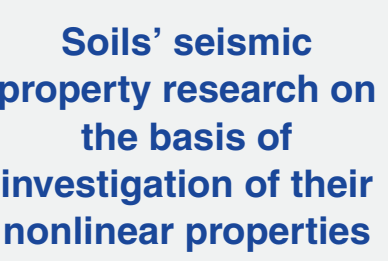

V. B. Zaalishvili
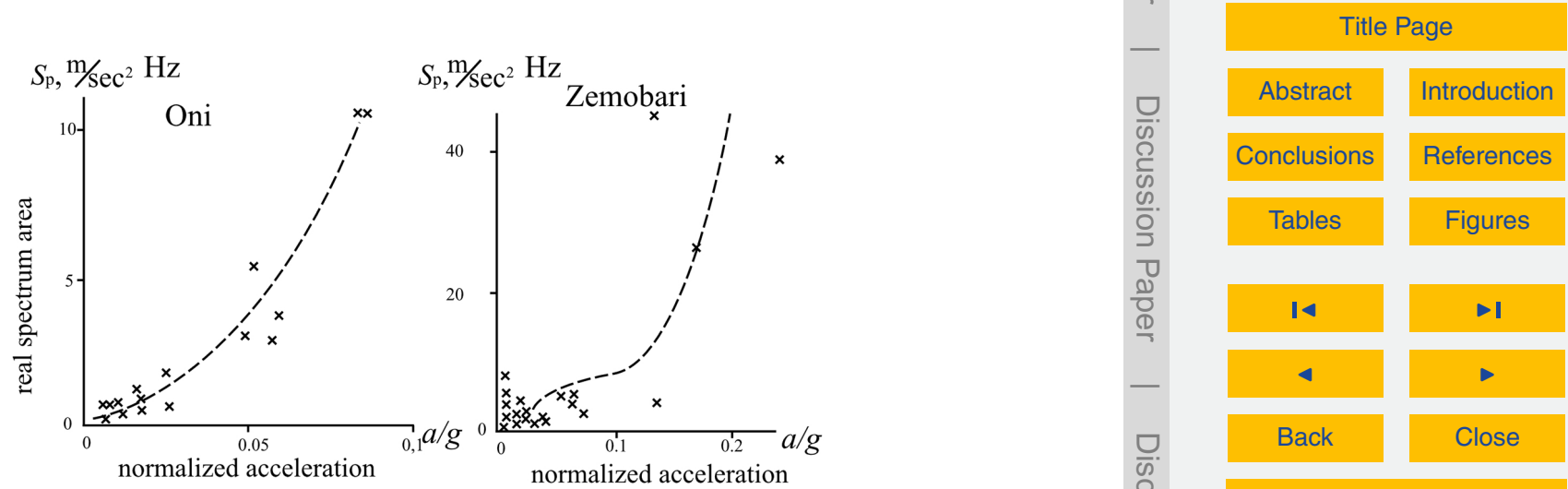

Full Screen / Esc

Figure 4. Indices of soil motion in the function of normalized acceleration $\mathrm{ag}^{-1}$ (Georgia, 1991).

\section{Printer-friendly Version}

Interactive Discussion 

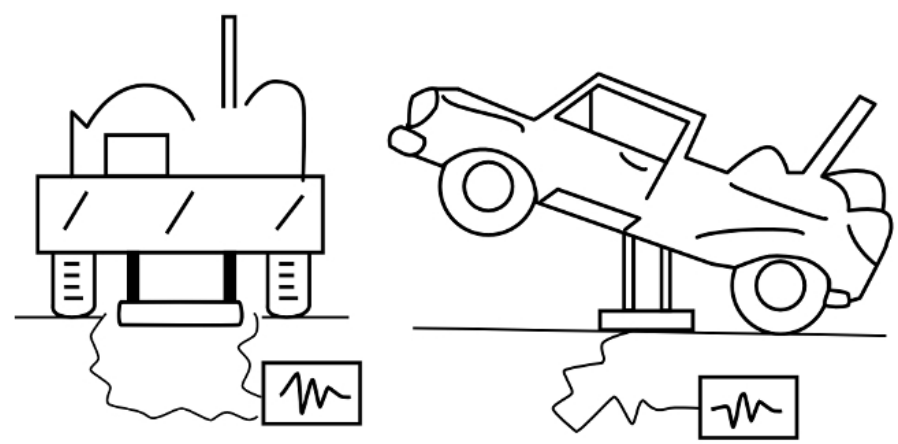

Figure 5. Determination of the nonlinearity in the form of strain sensitivity of soil.

\section{NPGD}

2, 425-474, 2015

Soils' seismic property research on the basis of investigation of their nonlinear properties

V. B. Zaalishvili

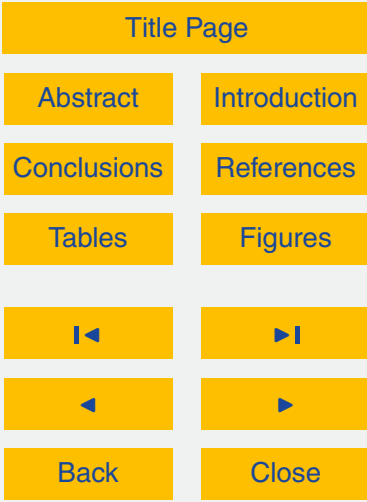

Full Screen / Esc

Printer-friendly Version

Interactive Discussion 


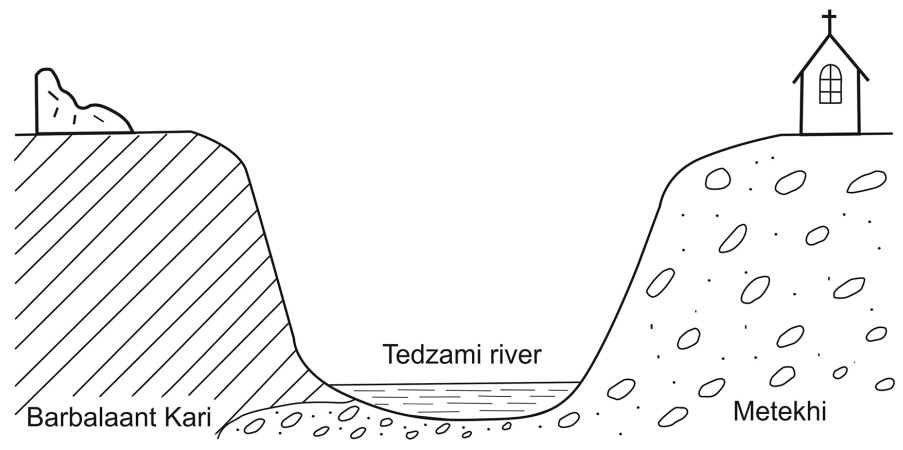

Figure 6. The areas with different intensity effects of the earthquake.

NPGD

2, 425-474, 2015

Soils' seismic property research on the basis of investigation of their nonlinear properties

V. B. Zaalishvili

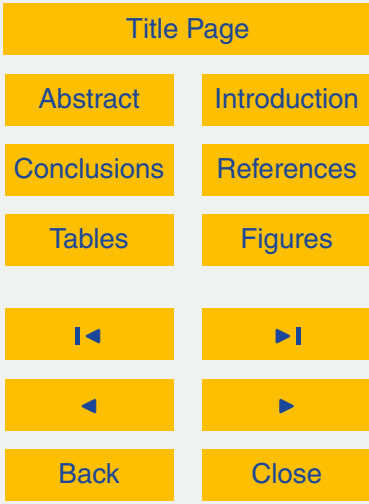

Full Screen / Esc

Printer-friendly Version

Interactive Discussion 


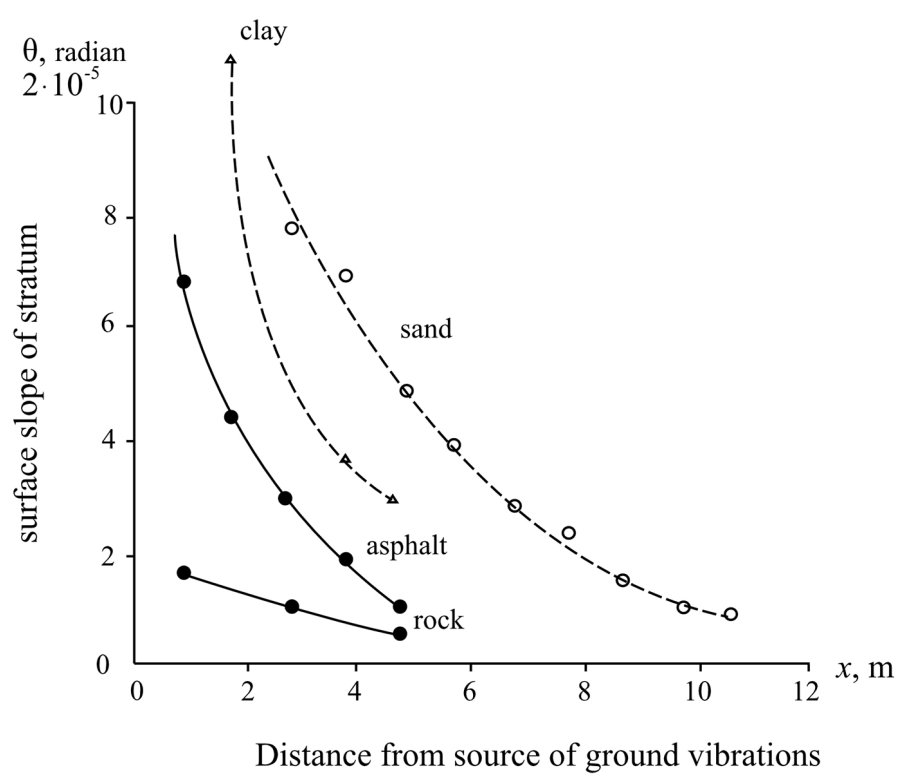

Figure 7. Dependence of the slope of the ground on the distance from the source (Uznozh, Belarus, 1992; Dedoplistskaro, Georgia, 1992).

\section{NPGD}

2, 425-474, 2015

Soils' seismic property research on the basis of investigation of their nonlinear properties

V. B. Zaalishvili

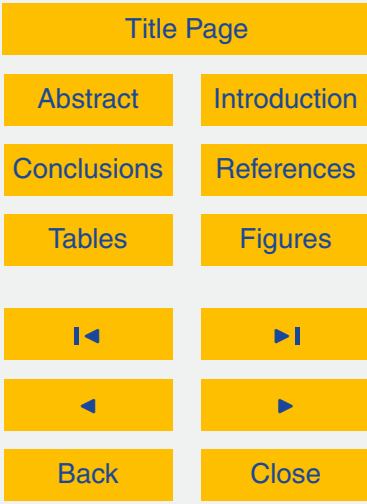

Full Screen / Esc

Printer-friendly Version

Interactive Discussion 


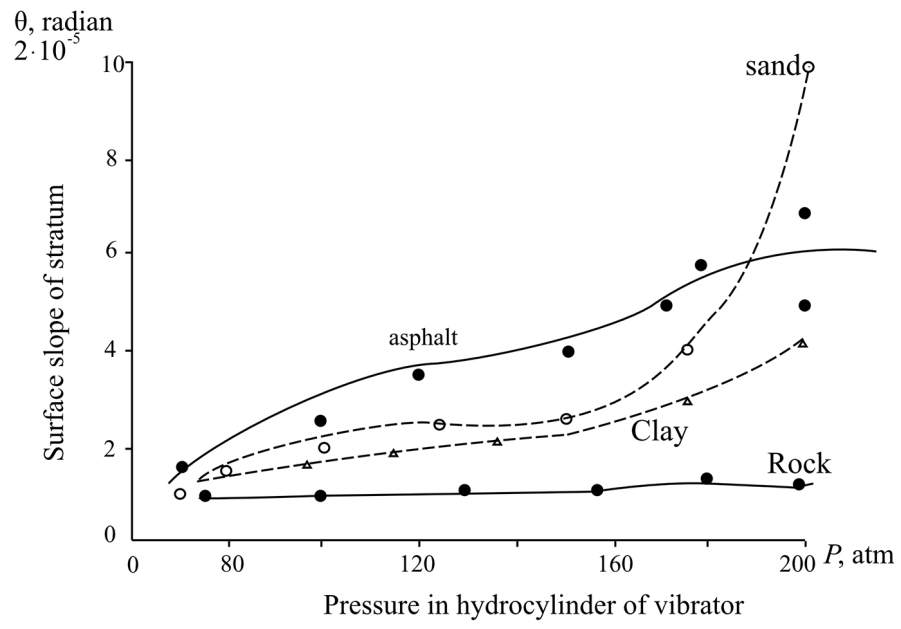

Figure 8. Influence of pressure in the hydraulic cylinder of the vibrator on the seismic radiating effect (Uznozh, Belarus, 1992; Dedoplistskaro, Georgia, 1992).

\section{NPGD}

2, 425-474, 2015

Soils' seismic property research on the basis of investigation of their nonlinear properties

V. B. Zaalishvili

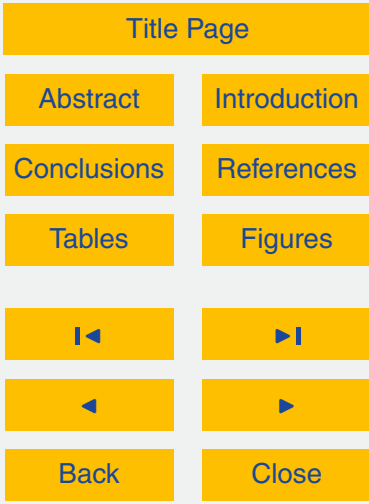

Full Screen / Esc

Printer-friendly Version

Interactive Discussion 

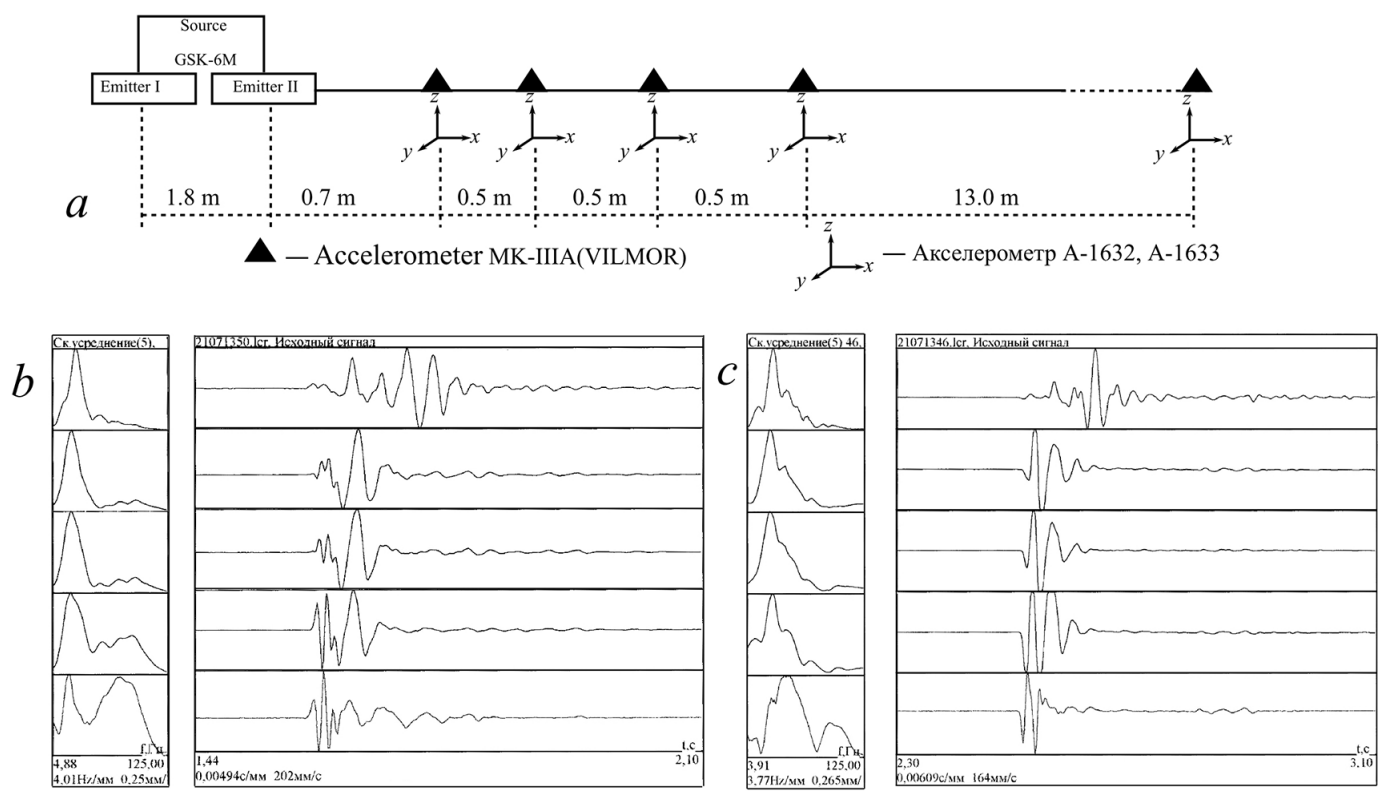

Figure 9. Investigations of spectral features from the source GSK-6M: (a) profile measurements of the area; (b) record of ground vibrations from the second hammer; (c) record of ground vibrations from the first hammer.

\section{NPGD}

2, 425-474, 2015

Soils' seismic property research on the basis of investigation of their nonlinear properties

V. B. Zaalishvili

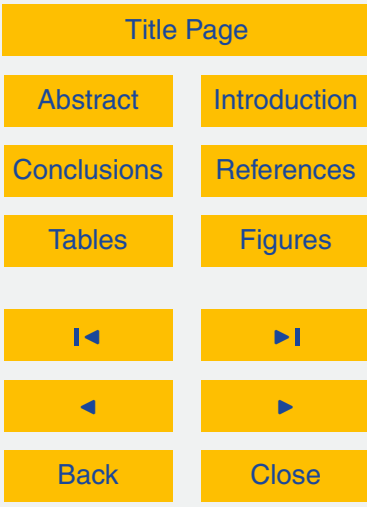

Full Screen / Esc

Printer-friendly Version

Interactive Discussion 


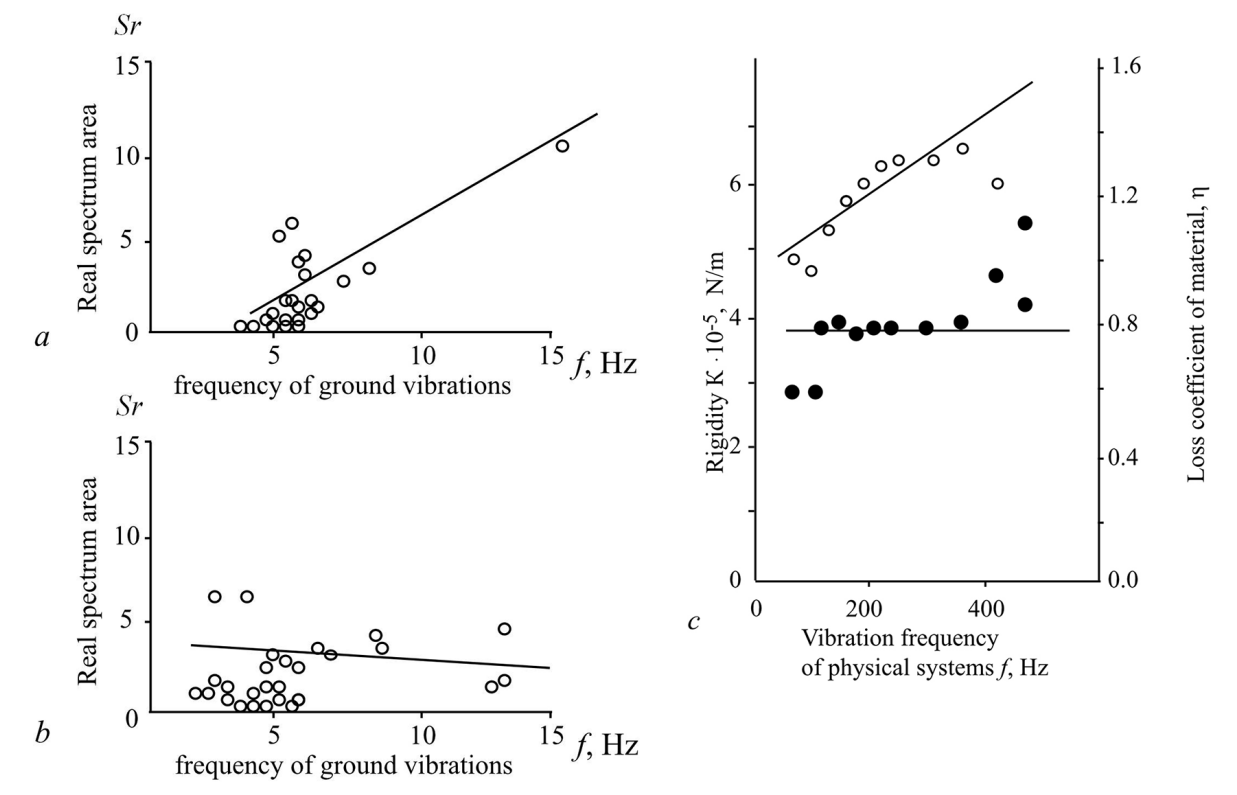

Figure 10. Dependence of real spectrum square of rocks ( $\mathbf{a}-$ Oni) and soft soils (b-Ambrolauri) on vibration frequency; dependence of rigidity and material loss coefficient on vibration frequency (c).

\section{NPGD}

2, 425-474, 2015

Soils' seismic property research on the basis of investigation of their nonlinear properties

V. B. Zaalishvili

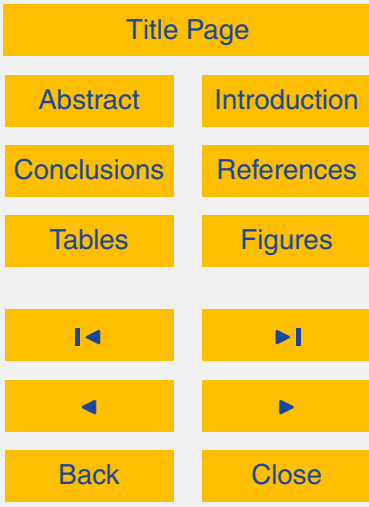

Full Screen / Esc

Printer-friendly Version

Interactive Discussion 


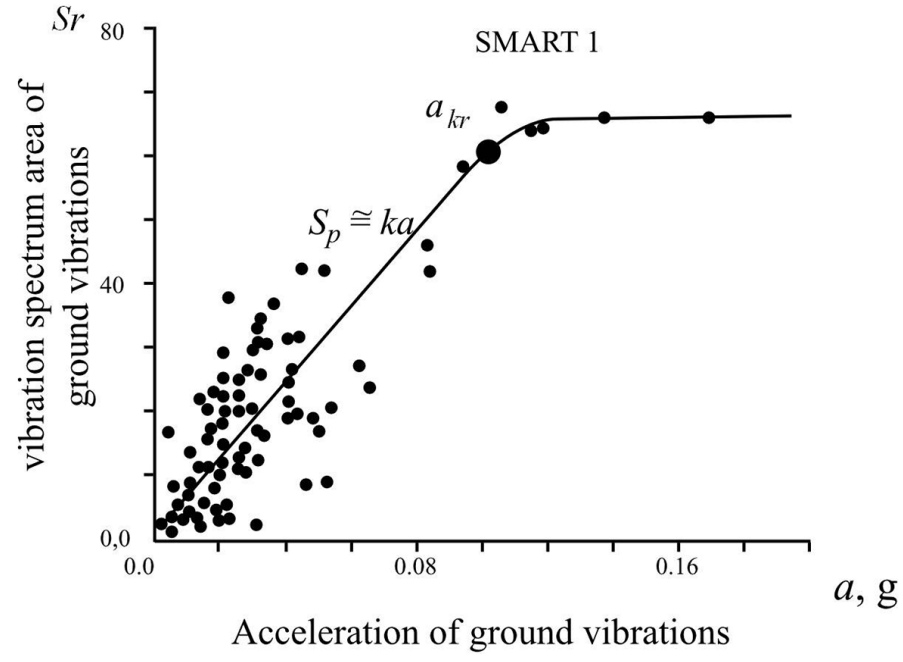

\section{NPGD}

2, 425-474, 2015

Soils' seismic property research on the basis of investigation of their nonlinear properties

V. B. Zaalishvili

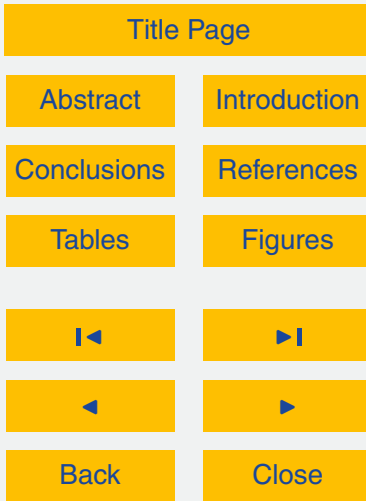

Full Screen / Esc

Printer-friendly Version

Interactive Discussion 


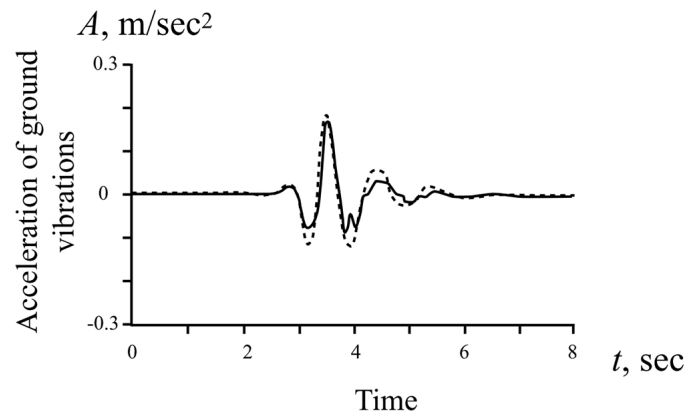

NPGD

2, 425-474, 2015

Soils' seismic property research on the basis of investigation of their nonlinear properties

V. B. Zaalishvili
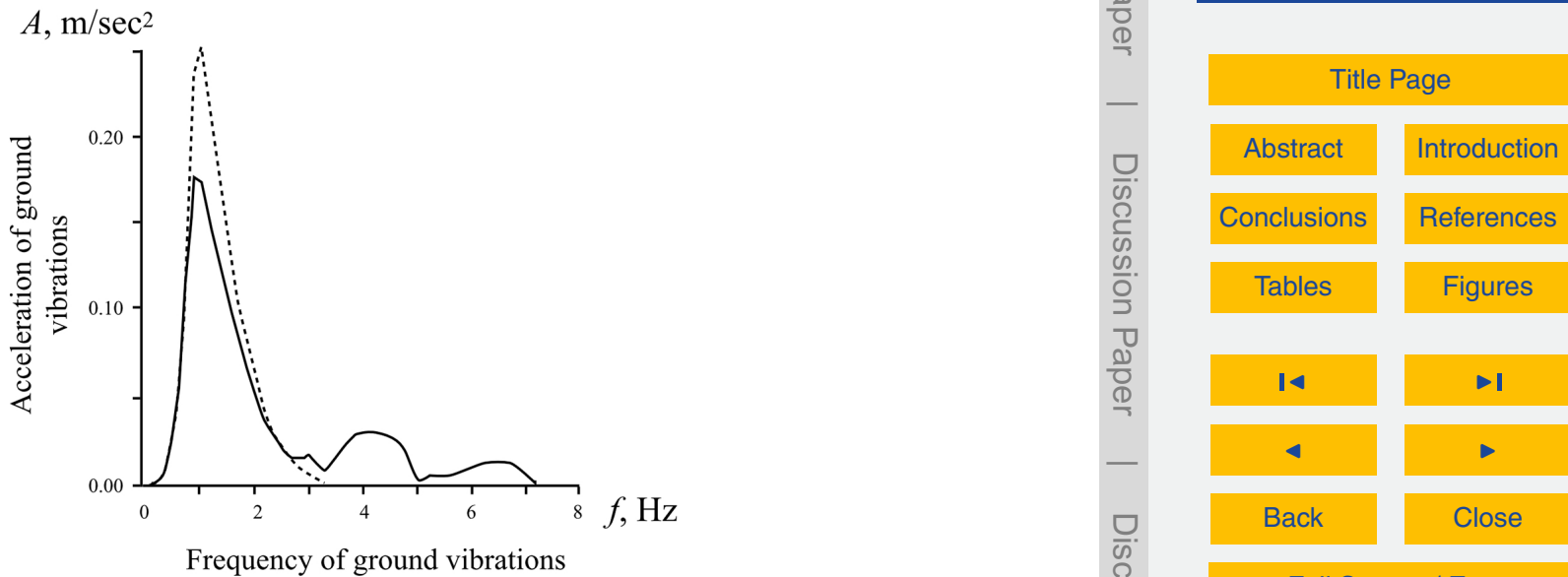

Full Screen / Esc

Figure 12. Comparison of the design accelerograms and corresponding Fourier spectra in linear and nonlinear solutions: the dotted line means a linear solution.

Printer-friendly Version

Interactive Discussion 


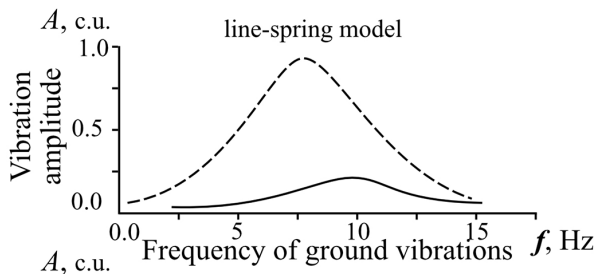

$\left.{ }^{1.0}\right] \quad$ line-spring model

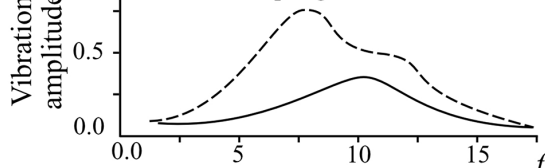

$A$, c.u. Frequency of ground vibrations $f, \mathrm{~Hz}$

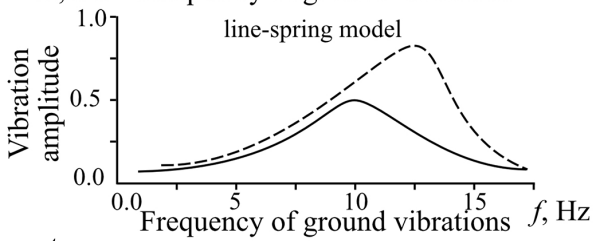
$A$, c.u.

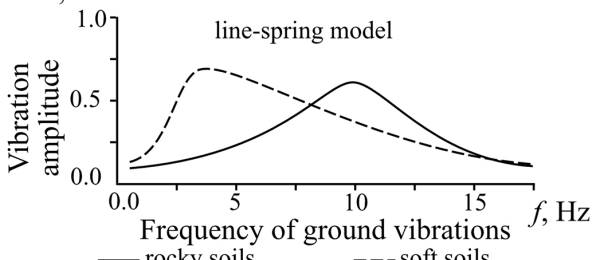

_ rocky soils _ - -- soft soils

Figure 13. Models of soil behavior on varying impacts.
NPGD

2, 425-474, 2015

Soils' seismic property research on

the basis of investigation of their nonlinear properties

V. B. Zaalishvili

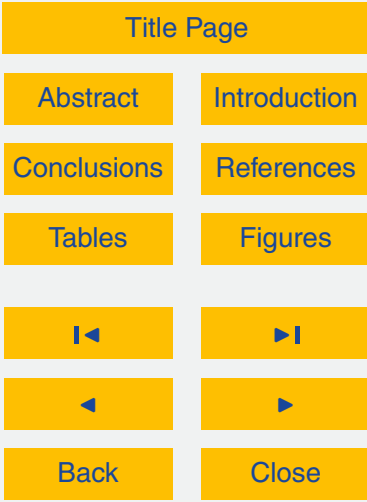

Full Screen / Esc

Printer-friendly Version

Interactive Discussion 


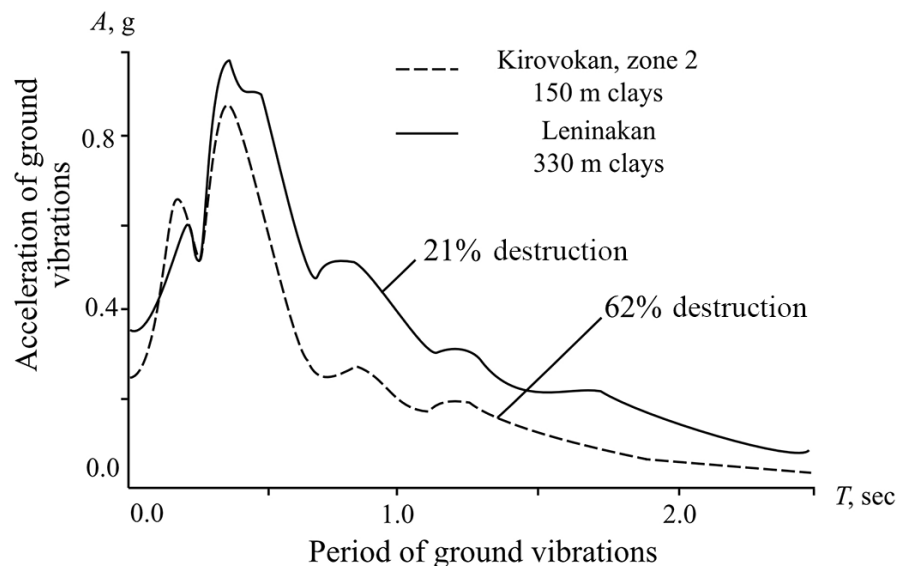

\section{NPGD}

2, 425-474, 2015

Soils' seismic property research on the basis of investigation of their nonlinear properties

V. B. Zaalishvili

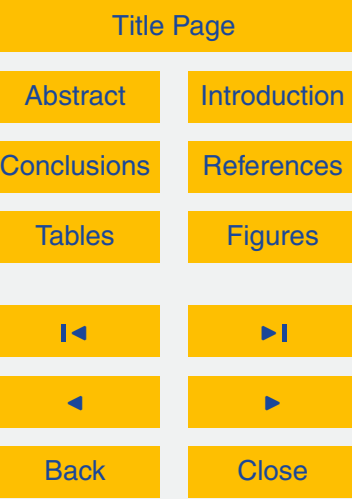

Full Screen / Esc

Printer-friendly Version

Interactive Discussion 